\title{
Time-Dependent Stresses From Fluid Extraction and Diffusion With Applications to Induced Seismicity
}

\author{
Valère Lambert ${ }^{1}$ \\ Seismological Laboratory, \\ California Institute of Technology, \\ Pasadena, CA 91125 \\ e-mail: vlambert@caltech.edu \\ Victor C. Tsai \\ Associate Professor \\ Department of Earth, Environmental \\ and Planetary Sciences, \\ Brown University, \\ Providence, RI 02912 \\ e-mail: victor_tsai@brown.edu
}

Over recent decades, it has become clear that the extraction of fluids from underground reservoirs can be linked to seismicity and aseismic deformation around producing fields. Using a simple model with uniform fluid extraction from a reservoir, Segall (1989, "Earthquakes Triggered by Fluid Extraction," Geology, 17(10), pp. 942-946) illustrated how poroelastic stresses resulting from fluid withdrawal may be consistent with earthquake focal mechanisms surrounding some producing fields. Since these stress fields depend on the spatial gradient of the change in pore fluid content within the reservoir, both quantitative and qualitative predictions of the stress changes surrounding a reservoir may be considerably affected by assumptions in the geometry and hydraulic properties of the producing zone. Here, we expand upon the work of Segall (1989, "Earthquakes Triggered by Fluid Extraction," Geology, 17, pp. 942-946 and 1985, "Stress and Subsidence Resulting From Subsurface Fluid Withdrawal in the Epicentral Region of the 1983 Coalinga Earthquake," J. Geophys. Res. Solid Earth, 90, pp. 6801-6816) to provide a quantitative analysis of the surrounding stresses resulting from fluid extraction and diffusion in a horizontal reservoir. In particular, when considering the diffusion of fluids, the spatial pattern and magnitude of imposed stresses is controlled by the ratio between the volumetric rate of fluid extraction and the reservoir diffusivity. Moreover, the effective reservoir length expands over time along with the diffusion front, predicting a time-dependent rotation of the induced principal stresses from relative tension to compression along the ends of the producing zone. This reversal in perturbed principal stress directions may manifest as a rotation in earthquake focal mechanisms or varied sensitivity to poroelastic triggering, depending upon the criticality of the pre-existing stress state and fault orientations, which may explain inferred rotations in principal stress directions associated with some induced seismicity. [DOI: 10.1115/1.4047034]

Keywords: computational mechanics, elasticity, stress analysis

\section{Introduction}

Induced seismicity is a topic of growing scientific interest and societal importance, particularly surrounding practices in resource extraction and waste disposal [1-4]. Many processes considered surrounding induced seismicity focus on solid-fluid interactions governing fault strength and stress. Much attention has been focused on the direct effects of fluid injection and increasing pore pressure in decreasing the effective normal stress across fault surfaces and potentially destabilizing faults under their preexisting stress conditions [3,5-17]. However, there is continued evidence for the presence of seismicity and aseismic deformation caused by the extraction of pore fluids, particularly in oil and gas fields where pore pressures may be declining by up to several 10 s of MPa [18-29].

Segall [30] proposed that the poroelastic stresses resulting from fluid withdrawal can be responsible for triggering earthquakes in producing fields on their own. A key aspect highlighted within his work is that the strain mismatch due to reservoir rocks contracting more than their surroundings generates stresses in areas where no changes in the pore-fluid content occur. Segall [30] produced stress field solutions encompassing a relatively simple reservoir

\footnotetext{
'Corresponding author

Contributed by the Applied Mechanics Division of ASME for publication in the Journal OF Applied Mechanics. Manuscript received January 20, 2020; final manuscript received April 20, 2020; published online May 14, 2020. Assoc. Editor: N.R. Aluru.
}

geometry assuming a uniform change in fluid mass content along a horizontal layer. The resulting fields illustrate that, as fluid is extracted, the overlying and underlying layers experience relative horizontal contraction and the neighboring regions on the flanks of the producing zone experience relative tension. Such solutions predict a preference for reverse faulting above and below the producing layer and normal faulting on the flanks of the producing zone, which is qualitatively consistent with observed seismicity around several producing fields (e.g., Goose Creek, Wilmington, Buena Vista Hills, Alberta, and Pau). One drawback of the solutions presented by Segall [30] is the existence of stress discontinuities at the edges of the reservoir due to the gradient in the change in fluid mass content being undefined at the boundaries of the producing region. Steep gradients in the change in the pore fluid content result in local stress concentrations, and the finiteness of the resulting solutions depend on the continuity of the fluid distributions [30,31]. As such, quantitative as well as qualitative predictions for the surrounding stresses caused by fluid extraction and injection may be substantially affected by basic assumptions in the geometry and hydraulic properties of the producing region.

The particular two-dimensional (2D) problem for fluid extraction from an infinite horizontal layer with uniform diffusivity was introduced by Segall [32], accounting for the smooth temporal evolution of the change in fluid content through diffusion. However, while considering the effects of varying diffusivity on surface subsidence, he mainly focused on induced stress changes well below the producing zone in order to estimate the stresses imposed on the 1983 Coalinga fault by oil field operations, which is several factors 
deeper than the corresponding production depth. Since seismicity can also often be observed in the layers overlying and surrounding the producing or injection zone, the focus in this study is to expand the analyses of Segall $[30,32]$ to examine the evolution of the stress field directly surrounding the producing zone in a reservoir including the smooth temporal evolution of the pore fluid distribution through diffusion. While the fixed reservoir model of Segall [30] was shown to qualitatively agree well with patterns in surface deformation and seismicity surrounding reservoirs with potentially comparable reservoir geometries, we note that the particular example of a laterally unconstrained reservoir with a finite diffusivity may be more quantitatively applicable to other producing fields where changes in fluid mass may not be considered uniform within the producing region (e.g., central Oklahoma [15], North and South Dakota [33], and Groningen [34]). Moreover, for many reservoir settings, the timescale for fluid transport (days to years) within the producing layer may be comparable to the timescale of field observations at relevant distances. For such cases, it may be important to account for the nonstationarity of the geometry of the effective producing zone when interpreting field measurements and seismological inferences [15,35,36]. For example, numerical models have illustrated how the temporal evolution of pore pressure and poroelastic stresses due to fluid injection and diffusion can help explain temporal trends in the productivity of triggered seismicity, depending on pre-existing fault geometries and background stress conditions [37].

The aim of this study is to provide an additional reference model that illustrates the potential importance of considering the nonstationarity of fluid sources when interpreting field observations, and from which exact calculations may be made for quantitative comparison with field data. This model may also serve as a benchmark problem for more complicated numerical studies. While more sophisticated numerical methods have been applied for detailed studies of some well-instrumented reservoirs in which the geometry and hydraulic properties are better constrained, we hope that these relatively simple solutions may prove useful in regions with limited reservoir data. In order to further facilitate such comparisons, we provide two potential approximate extensions to 3D for relevant production quantities such as the volumetric flowrate. In addition, we briefly discuss the implications of our model results for existing field observations and seismological studies, including inferred temporal variations in the principal stress directions of seismic events around injection wells [38-40].

\section{Model Description}

Segall [32] showed that for a linear, isotropic poroelastic medium, the solid displacements $u_{j}$ due to distributed changes in fluid mass content $\Delta m(\mathbf{y}, t)$ can be expressed through a linear integral relationship over the source volume $V_{\mathbf{y}}$ as

$$
u_{j}(\mathbf{x}, t)=\frac{\left(1+\nu_{u}\right) B}{3 \pi \rho_{0}\left(1-\nu_{u}\right)} \int_{V} \Delta m(\mathbf{y}, t) g_{j}(\mathbf{x}, \mathbf{y}) d V_{\mathbf{y}}
$$

where $\nu_{u}$ is undrained Poisson's ratio, $B$ is Skempton's pore pressure coefficient, and $\rho_{0}$ is the fluid density in the reference state. The function $g_{j}(\mathbf{x}, \mathbf{y})$ represents the displacement in the $j$ direction at $\mathbf{x}$ due to a point center of dilatation at $\mathbf{y}$ with associated change in fluid mass content $\Delta m(\mathbf{y}, t) d V$. Note that $\mathbf{y}$ and $\mathbf{x}$ denote the full position vectors for the source and receiver elements, respectively. The total displacement $u_{j}(\mathbf{x}, t)$ due to quasi-static changes in a distributed fluid mass at time $t$ is then obtained by integrating the undrained point fluid mass changes within the region for which $\Delta m(\mathbf{y}, t)$ is non-zero.

The change in the fluid mass content is defined as the change in fluid mass per unit solid volume and can be related to the mean solid stress $\sigma_{k k}$ and pore fluid pressure $p$ as

$$
\Delta m=\frac{\left(1-2 \nu_{u}\right) \alpha \rho_{0}}{2 \mu\left(1+\nu_{u}\right)}\left[\sigma_{k k}+\frac{3}{B} p\right]
$$

where $\alpha$ relates the bulk modulus of the fluid-saturated rock under drained conditions, $K$, and that of the solid rock, $K_{s}$, as $\alpha=1-K / K_{s}$ [41]. Stresses may be related to the displacements and associated strains through the linear isotropic poroelastic constitutive relationship

$$
2 \mu \epsilon_{i j}=\sigma_{i j}-\frac{\nu_{u}}{1+\nu_{u}} \sigma_{k k} \delta_{i j}+\frac{2 \mu B}{3 \rho_{0}} \Delta m \delta_{i j}
$$

such that the stress distribution due to the integrated source is given by

$$
\sigma_{i j}(\mathbf{x}, t)=\frac{\mu\left(1+\nu_{u}\right) B}{3 \pi \rho_{0}} \int_{V} \Delta m(\mathbf{y}, t) G_{i j}(\mathbf{x}, \mathbf{y}) d V_{\mathbf{y}}-\frac{B K_{u}}{\rho_{0}} \Delta m(\mathbf{y}, t) \delta_{i j}
$$

where $\mu$ is the shear modulus and $K_{u}$ is the undrained bulk modulus [32]. Solutions for displacements and stresses due to the change in fluid mass within a distributed volume can therefore be represented through integral expressions of the prescribed change in the fluid mass content and the poroelastostatic Green's functions $G_{i j}(\mathbf{x}, \mathbf{y})$ and $g_{j}(\mathbf{x}, \mathbf{y})$ which have been presented by Segall [32] and included within the Appendix. Note that in this work, we express solutions for displacements and stresses due to changes in the fluid mass content and do not attempt to solve for the associated pore pressures within the reservoir. However, pore pressure distributions within the producing zone may be calculated from the change in pore fluid mass and the associated solutions for the mean solid stress through Eq. (2) [32].

Models for the Fluid Mass Distribution. Segall [30] examined the $2 \mathrm{D}$ poroelastic stress field and surface displacements caused by fluid extraction from a horizontal, permeable layer of thickness $T$, which was assumed small compared to the reservoir depth $D$, and enclosed in an otherwise impermeable half-space (Fig. 1(a)). $\Delta m(\mathbf{y}, t)$ is considered uniform over a horizontal interval $-a<y_{1}$ $<a$, as well as with depth $D<y_{2}<D+T$, reflecting a producing layer of fixed length $2 a$ and thickness $T$. The specific case where $a=D$ was examined by Segall [30], which we also consider in this study for direct comparison. We set the net mass flux out of the producing zone $-Q$ to be constant for time $t>0$ and zero for $t$ $<0$, and thus can express this uniform change in the fluid mass distribution as a function of the mass flux and time simply as

$$
\Delta m_{f}(\mathbf{x}, t)=-\frac{Q t}{2 a} B\left(x_{1} ;-a, a\right) B\left(x_{2} ; D, D+T\right)
$$

where $B\left(x ; \zeta_{1}, \zeta_{2}\right)$ refers to the boxcar function over $x$ from $\zeta_{1}$ and $\zeta_{2}$. We denote this mass distribution as $\Delta m_{f}(\mathbf{x}, t)$ and will henceforth refer to it as the fixed reservoir model. Note that $Q$ reflects the change in fluid mass per unit time and cross-sectional area transverse to $x_{1}$, considering a uniform distribution over $T$ and an additional length scale $W$ not explicitly modeled here out-of-plane along $x_{3}$. Two key features of this model are that the reservoir geometry is fixed with length $2 a$ and the temporal evolution of the fluid mass distribution neglects the finite timescale for fluid migration toward the extraction site at $x_{1}=0$. In essence, the fixed reservoir model reflects extraction from a geometrically constrained reservoir with effectively infinite internal diffusivity, allowing for instantaneous equilibration of the fluid mass distribution upon further fluid extraction or injection.

In order to account for the redistribution of fluid mass due to diffusion, we consider the case introduced by Segall [32] of an infinite horizontal layer of thickness $T$ and diffusivity $c$, similarly buried at a depth of $D$ in an otherwise impermeable medium. The spatiotemporal evolution of the fluid mass distribution within the infinite 
(a)

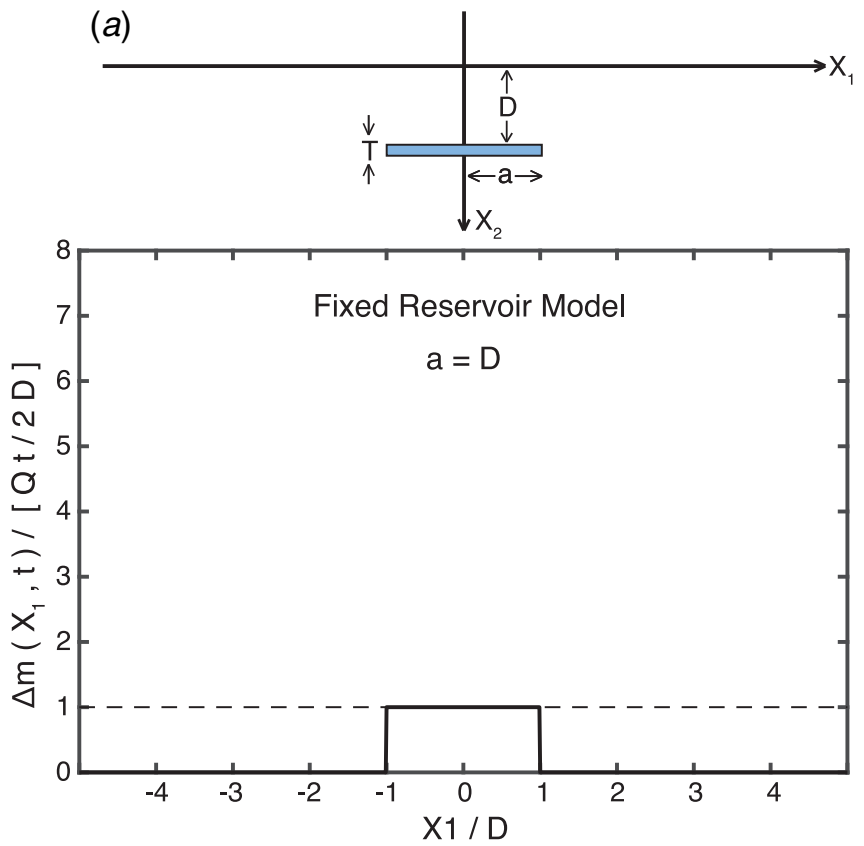

(b)
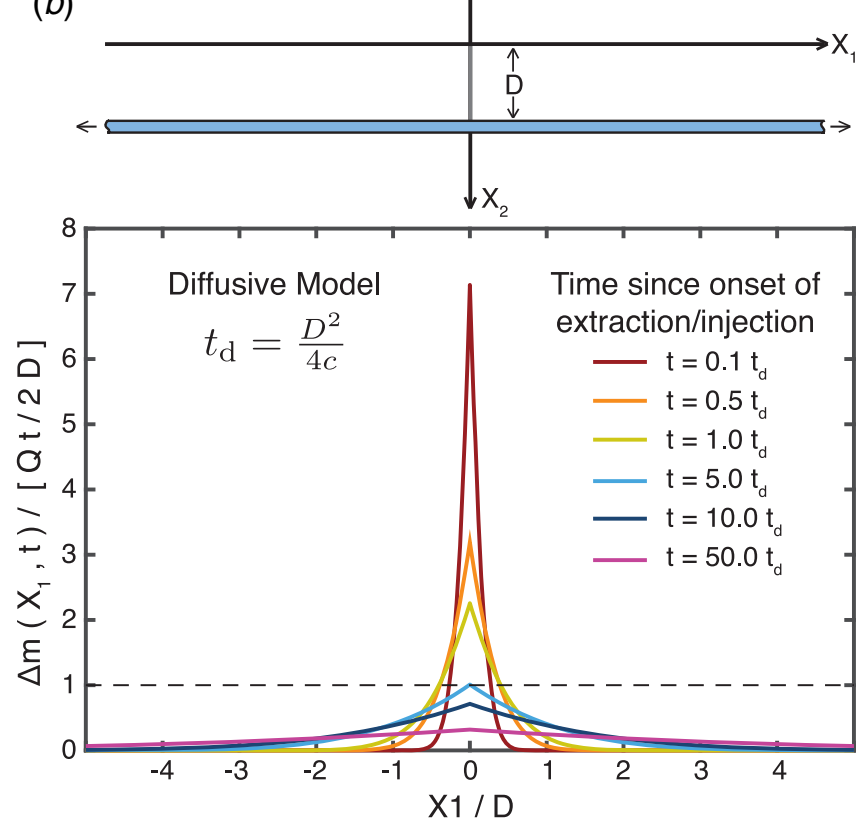

Fig. 1 Model geometry (top) and distributions for the change in fluid mass content (bottom) for the fixed reservoir model (a) as described in Ref. [30] and the diffusive reservoir model (b) as described in Ref. [32]. The half-width of the fixed reservoir is assumed to be equal to the reservoir depth. Fluid mass content distributions for the diffusive reservoir model are shown at increasing times with relation to the characteristic diffusion timescale, $t_{d}$. The spatial distributions of the fluid mass change are scaled by the total mass content divided by twice the reservoir depth.

layer is then governed by the one-dimensional diffusion equation

$$
\frac{\partial \Delta m(\mathbf{x}, t)}{\partial t}=c \frac{\partial^{2} \Delta m(\mathbf{x}, t)}{\partial x_{1}^{2}}
$$

with the local fluid mass flux $q$ subject to the boundary conditions

$$
\begin{aligned}
& q\left(x_{1}=0^{+}\right)-q\left(x_{1}=0^{-}\right)=-Q, t>0 \\
& q\left(x_{1}= \pm \infty\right)=0, t>0
\end{aligned}
$$

Accounting for diffusion with respect to the plane at $x_{1}=0$, the fluid mass change per unit solid volume can be expressed as [42]

$$
\Delta m_{d}(\mathbf{x}, t)=-Q\left(\frac{t}{c}\right)^{1 / 2} \operatorname{ierfc}\left[\left(\frac{x_{1}^{2}}{4 c t}\right)^{1 / 2}\right] B\left(x_{2} ; D, D+T\right)
$$

for $t>0$, where ierfc $[x]=\int_{0}^{x} \operatorname{erfc}[x] d x=e^{-x^{2}} / \pi^{1 / 2}-x \operatorname{erfc}[x]$ is the first integral of the complementary error function. Here, we denote the mass distribution accounting for fluid diffusion as $\Delta m_{d}(\mathbf{x}, t)$ and refer to it as the diffusive model (Fig. 1(b)).

In contrast to the fixed reservoir model given by Eq. (5), the diffusive model does not contain a fixed reservoir length scale. Instead one can consider an effective producing zone length approximately equal to the diffusion length $l_{d}=\sqrt{4 c t}$, which describes the spatial extent to which the change in fluid mass concentration due to extraction has propagated with time. Note here that $T$ is assumed small compared to $D$ and $l_{d}$ such that the mass distribution is considered uniform with depth inside the reservoir. This assumption is that $T<l_{d}$ breaks down as $t \rightarrow 0$ during the onset of fluid extraction. However, we will primarily focus on timescales where $t$ approaches or is greater than a reference diffusion timescale, $t_{d}=$ $D^{2} / 4 c$, based on the reservoir depth, and thus where $l_{d} \gtrsim D>T$, such that this approximation is more appropriate. Assuming a reservoir depth of $1 \mathrm{~km}$, values for $t_{d}$ would be around 2.9 days after the onset of extraction for a hydraulic diffusivity of $1 \mathrm{~m}^{2} / \mathrm{s}$ and 29 days for a diffusivity of $0.1 \mathrm{~m}^{2} / \mathrm{s}$, which represent moderate values of relatively high and low diffusivities for production fields, respectively [32]. Since the diffusion length scale increases with the square root of time, $l_{d}$ approaches $10 \%$ of $D$ when $t$ is only at $1 \%$ of $t_{d}$, meaning that the effective reservoir length is within one order of magnitude of the reservoir depth much earlier than the corresponding reference diffusion timescale.

In considering only the stress distribution outside of the reservoir, where $\Delta m(\mathbf{x}, t)=0$, Eq. (4) reduces to

$$
\sigma_{i j}(\mathbf{x}, t)=C \int_{V} \Delta m(\mathbf{y}, t) G_{i j}(\mathbf{x}, \mathbf{y}) d V_{\mathbf{y}}
$$

where $C=\mu\left(1+v_{u}\right) B / 3 \pi \rho_{0}\left(1-v_{u}\right)$, such that the stresses in the fixed reservoir model (Eq. (5)) are

$$
\sigma_{i j}(\mathbf{x}, t)=\frac{C Q t}{2} \int_{V} G_{i j}(\mathbf{x}, \mathbf{y}) d V_{\mathrm{y}}
$$

and those for the diffusive model (Eq. (7)) are

$$
\sigma_{i j}(\mathbf{x}, t)=C Q\left(\frac{t}{c}\right)^{1 / 2} \int_{V} \operatorname{ierfc}\left[\left(\frac{y_{1}^{2}}{4 c t}\right)^{1 / 2}\right] G_{i j}(\mathbf{x}, \mathbf{y}) d V_{\mathbf{y}}
$$

Full expressions for the stresses and displacements for both distributions are presented in the Appendix where full closed form solutions may be expressed for the fixed reservoir model while the diffusive model includes an integral over the horizontal layer which may be computed numerically.

An important distinction between Eqs. (9) and (10) is the temporal dependence of the stress fields. The fixed reservoir solutions are separable in space and time such that while the total change in the fluid mass content increases over time, it is always evenly distributed over the reservoir length $2 a$, thereby increasing the magnitude of the same spatial distribution. For the diffusive case, the spatial distribution of the fluid mass is coupled to the temporal evolution with respect to the inception of extraction or injection. In such a manner, as the total change in the fluid content increases over time, the spatial extent over which this contribution is distributed also grows with the effective diffusion length scale $l_{d}$, such that the concentration of the change in the fluid mass content is not geometrically fixed throughout time. We explore the implications of the 
spatio-temporal evolution of the effective producing zone in the "Results" section.

\section{Results}

We consider the cases of fluid extraction from a single site in both the fixed and diffusive reservoir models and examine the spatial distributions of the displacements and stresses surrounding the reservoirs, as well as the temporal evolution of these fields in the case of the diffusive model. Figure 2 compares the surface displacements and horizontal strain as well as the horizontal normal stress $\sigma_{11}$ and maximum shear stress fields $\left(\tau=\left[\left(\sigma_{11}-\sigma_{22}\right)^{2} / 4+\sigma_{12}^{2}\right]^{1 / 2}\right)$ caused by fluid extraction (stresses positive in tension) from the fixed (Fig. 2(a)) and diffusive (Figs. 2(b)-2(d)) reservoir models. The results for the fixed model are the same as those presented by Ref. [30]. Three cases are shown for the diffusive model with hydraulic diffusivities varying by an order of magnitude $c=0.1$ $c_{D}, c=c_{D}$, and $c=10 c_{D}$ about the reference diffusivity $c_{D}$. Each diffusive case is calculated at the same time $t=t_{d}=D^{2} / 4 c_{D}$. All four examples are scaled to represent instances where the total extracted fluid volume is the same, but the spatial extent and concentration varies based on the reservoir geometry and diffusivity. Simulated focal mechanisms in each case illustrate planes of maximum shear stress $\left(\phi=\frac{1}{2} \tan ^{-1}\left[\left(\sigma_{22}-\sigma_{11}\right) /\left(2 \sigma_{12}\right)\right]\right)$ with black regions denoting quadrants of relative tension and white for quadrants of relative compression, with respect to each representative point. The spatial distribution of the surface displacements and extensional strain are qualitatively similar among the fixed and diffusive models shown in Fig. 2, but they differ quantitatively in both magnitude and localization, reflecting the intensity of the gradient in the change in fluid mass within each reservoir. As pointed out by Segall [32], the surface expressions depend strongly on the hydraulic diffusivity with more pronounced subsidence for lower diffusivities and relatively small and broadly dispersed subsidence for high diffusivities. Solutions for the fixed reservoir model are most similar to the diffusive model in Fig. 2(c) which has a similar effective producing zone half-length of $l_{d}=D$; however, vertical displacements are $8 \%$ more pronounced near the extraction site at $x_{1}=0$ for the diffusive model along with a $32 \%$ increase in the horizontal extensional strain (see Figs. $2(a)$ and $2(c)$ ). Note that the relative contraction and broadening of the surface expressions for the cases where $c=0.1 c_{D}$ and $c=10 c_{D}$, respectively, are also consistent with the time-dependent evolution of the effective reservoir length.

Geometric and Temporal Dependence of Stress Concentrations. An important conclusion about the reservoir geometry is that the intensity of imposed stresses due to injection or extraction depends on the spatial gradient of the change in fluid mass content, such that higher stress concentrations are located around regions with sharper gradients in $\Delta m$. This is evident in the fixed reservoir model where discontinuities in the fluid mass distribution at the ends of the producing zone introduce large stress concentrations near the edges of the reservoir (Fig. 2(a)). In the case of the diffusive model, a stress concentration is focused about the extraction site at $x_{1}=0$ due to the localized extraction of fluid, whereas the smooth decay of the distribution away from this point precludes the concentration of stresses elsewhere (Figs. 2(b)-2(d)). This stress concentration at $x_{1}=0$ is not present in the uniform model as the fluid distribution is assumed to equilibrate rapidly to provide a uniform change in fluid mass content across the reservoir.

In the diffusive model, the intensity of this stress concentration near the extraction point at any given time is a function of the rate of fluid extraction and the reservoir diffusivity, and is governed by the ratio of the volume flux into or out of the reservoir to the rate at which fluid can diffuse away or toward the loading point. For lower diffusivities, the same change in fluid mass is more sharply localized near the injection or extraction point resulting in more concentrated stresses (Fig. 2(b)). For higher diffusivities, sharp gradients in the change in fluid mass are alleviated more rapidly, thereby mitigating stress concentrations and resulting in smoother stress distributions (Fig. 2(d)). This emphasizes the significance of the reservoir diffusivity in determining the rate at which concentrated stresses induced by fluid extraction or injection can be relieved.

A more intuitive relationship may be made in terms of the volumetric rate of fluid extraction $\dot{V}$. We consider two simple approximations to extend our 2D model results to three dimension (3D). For case 1, we assume a fixed producing zone width $W$ in the $x_{3}$ direction, and in case $2, W$ is assumed to scale with the producing zone length in time. We consider the mass flux per effective producing zone length defined by the diffusion length scale, $Q / 4 \sqrt{c t}=\dot{M} / A T$, where $\dot{M}$ is the average rate of total fluid mass extraction and $A$ is the horizontal area of the producing zone in the $x_{1} \times x_{3}$ plane. In case 1 , the area can be described as $A=$ $4 \sqrt{c t} W$ with the volumetric rate of extraction $\dot{V}=\dot{M} / \rho_{0}$, giving the relation $Q / \rho_{0}=\dot{V} / W T$ for the effective volume flux, as in Ref. [32]. Alternatively, in case 2, the producing zone width may be considered to scale with the length through time as $W=\gamma \sqrt{c t}$ for $t>0$, where $\gamma$ is a positive constant. The area then is given by $A=4 \gamma c t$ and the effective volume flux is $Q / \rho_{0}=\dot{V} / \pi \gamma \sqrt{c t} T$. Accordingly, the prefactor $\left(1+\nu_{u}\right) B Q / 3 \pi \rho_{0} \sqrt{t / c}$ for the displacements and stresses associated with the diffusive model becomes $\left(1+\nu_{u}\right) B \dot{V} / 3 \pi W T \sqrt{t / c}$ for case 1 and $\left(1+\nu_{u}\right) B \dot{V} / 3 \pi^{2} \gamma c T$ for case 2. Here, the trade-off between the volumetric rate of fluid extraction and the diffusivity of the reservoir becomes more apparent, particularly in case 2 where the assumed linear increase in extracted fluid volume with time is compensated exactly by the linear increase in the effective producing zone volume. In this case, the prefactor has no temporal dependence, but the spatial distribution of the deformation and stress fields are still coupled to the temporal evolution of the effective producing zone volume. While the $2 \mathrm{D}$ solutions are exact, these extensions to 3D provide only crude approximations meant to facilitate quantitative comparison with field measurements. The solutions for the special case of an exactly axisymmetric mass distribution have been introduced by Segall [31].

Figure 3 shows the temporal evolution of the spatial distributions of the horizontal normal stress and maximum shear stress in the diffusive reservoir model throughout time. Stresses are normalized by $\left(\mu\left(1+v_{u}\right) B T / 6 \pi \rho_{0} D\left(1-v_{u}\right)\right)(Q t / \sqrt{4 c t})$ to reflect the balance between the increasing change in total extracted fluid volume, as well as the increase in the effective producing zone volume with the extension of the diffusion length scale. This normalization factor can also be replaced with the approximate 3D prefactors introduced in the previous section. In particular for case 2, this normalization coefficient becomes $\mu\left(1+v_{u}\right) B \dot{V} / 12 \pi^{2}\left(1-v_{u}\right) \gamma c D$ such that the scaling is independent of time and the amplitude depends linearly on the ratio of $V$ and $c$. We see that the stress concentration near the extraction site decreases as the producing zone expands and $\Delta m$ becomes smoother and more evenly distributed. Additional distributions of the horizontal and vertical normal stresses as well as the shear stresses at similar points in time for the diffusive model are shown in Fig. 4.

Rotation of Principal Stress Directions. A notable feature in Figs. 2 and 3 is that the orientation of the maximum shear stress at a given location, illustrated by the simulated focal mechanisms, is not static but rotates over time as the reach of the diffusion front advances. For example, in Fig. 3, the simulated mechanisms at the outer edges for both $x_{1}= \pm 3.5 \mathrm{D}$ and $x_{2}=0.25 \mathrm{D}$ rotate from horizontal relative tension to compression between the panels for $t=10 t_{d}$ and $t=100 t_{d}$. The temporal evolution of the angle of maximum extensional stress $\theta$ and angle of maximum shear stress $\phi$ is shown in Fig. 5 as functions of the lateral distance from the extraction axis at representative depths of $0.25 \mathrm{D}$ and 0.5 $D$. As the front of the producing zone expands away from the 

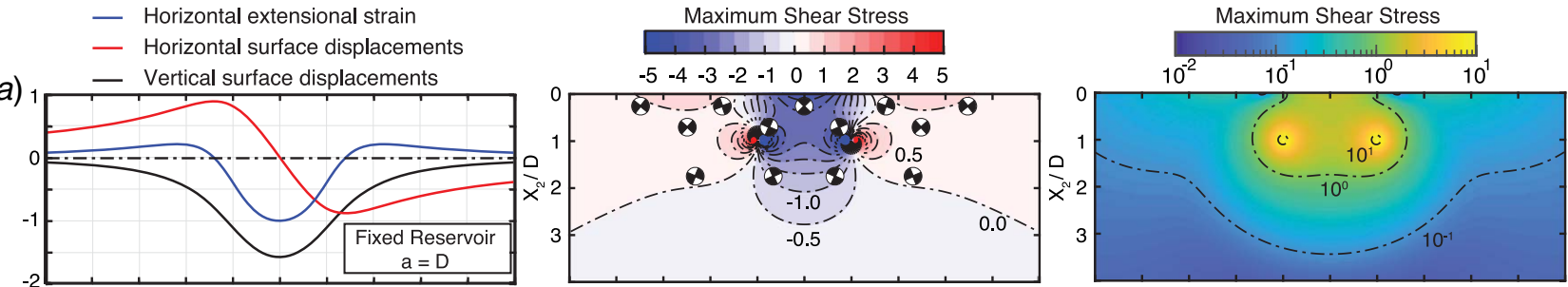

(b)
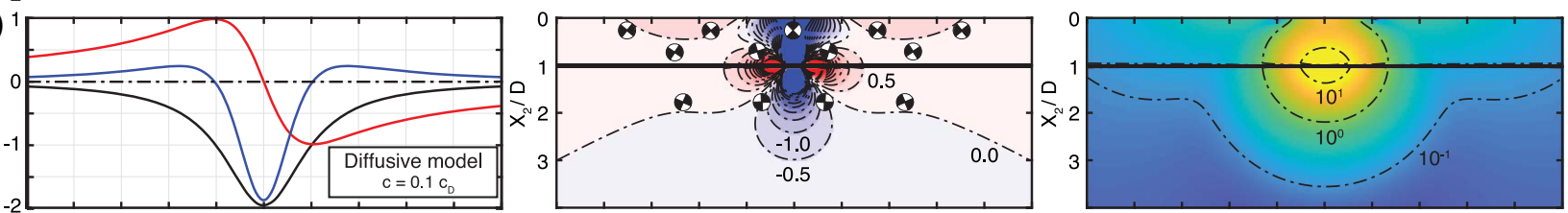

(c)
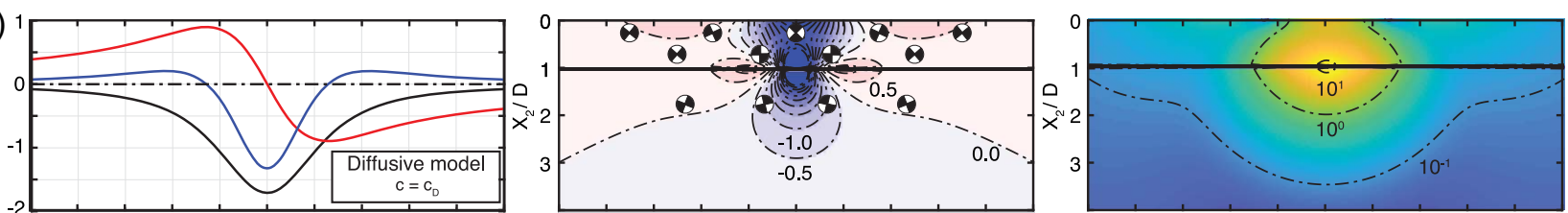

(d)
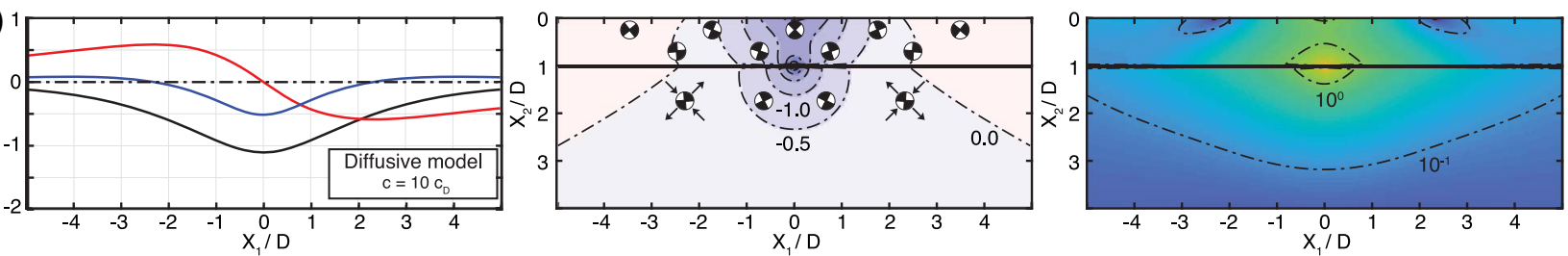

Fig. 2 Calculated displacements and horizontal extensional strain at the surface (left), change in horizontal normal stress (center) and maximum shear stress (right) due to fluid extraction (relative tension positive) in both the (a) box-car model and (b)-(d) diffusion models for varying hydraulic diffusivity with respect to $c_{D}$ at time $t=t_{d}$, where $t_{d}=D^{2} / 4 c_{D}$. Note that the convention for $x_{2}$ is switched for vertical surface displacements such that negative displacements reflect subsidence. Stresses are contoured outside the producing layer for the diffusive models $\left(x_{2} \in[D, D+T]\right)$. Simulated focal mechanisms indicate planes of maximum shear stress, with arrows indicating the axes of maximum tension and compression. Displacements are normalized by $\left(1+v_{u}\right)$ $B T Q t / 3 \pi \rho_{0} D$, strains by $\left(1+v_{u}\right) B T Q t / 3 \pi \rho_{0} D^{2}$ and stresses by $\mu\left(1+v_{u}\right) B T Q t / 6 \pi \rho_{0} D^{2}\left(1-v_{u}\right)$.

extraction point, the angle of maximum extensional stress change rotates such that points further away from the extraction site experience a reversal from relative horizontal tension to compression. The angle of maximum shear stress correspondingly rotates from around $-\pi / 4$ to $\pi / 4$, indicative of a transition from preferred normal faulting to reverse faulting. This is generally consistent with the trend of expected reverse faulting above and below the producing layer and normal faulting at the flanks of the producing layer, as discussed by Segall [30]. However, as the spatial extent of the producing zone grows with time, the preferred mechanism of seismicity at a given location may evolve as the surrounding region transitions into a state of compression. The temporal evolution of the magnitude and direction of the stresses at the locations highlighted in Fig. 5 are included in the Appendix (see Figs. 7 and 8).

Due to the interaction of the stress field with the free surface, the timing of the stress rotations varies slightly with depth but may be estimated given knowledge of the reservoir depth and diffusivity, as well as the depth and distance from the extraction site for the location of interest (Fig. 6). For a depth of interest around one-half of the reservoir depth, the timescale about which one would expect a transition to relative horizontal compression would be $t \approx t_{d}$ and $t \approx 5.6$ $t_{d}$ for lateral locations of $x_{1}=D$ and $x_{1}=2 D$, respectively. Considering a reservoir at $1 \mathrm{~km}$ depth and a diffusivity of $c=1 \mathrm{~m}^{2} / \mathrm{s}$, this gives approximate timescales for stress reversal between 1 and 2 $\mathrm{km}$ from the extraction site of approximately 3-16 days following initiation of extraction. For lower diffusivities, this window can be much broader. For instance, with a diffusivity of $c=0.1 \mathrm{~m}^{2} / \mathrm{s}$ for otherwise similar considerations, the time window for stress reversal between 1 and $2 \mathrm{~km}$ from the extraction site becomes roughly 29-145 days, potentially providing a more substantial observation window.
Depending on how the reservoir area scales with time, the magnitude of the stress perturbations may be expected to grow as fluid is continuously extracted. Therefore, the significance of the stress reversal depends on the diffusivity of the reservoir, as well as the ambient stress conditions before extraction. For a given time since initiation of pumping, the orientation of the stress perturbation depends on the diffusivity of the reservoir (Fig. 2). For reservoirs with higher diffusivities, locations surrounding the extraction site may experience the rotation in the perturbed stress before the perturbations achieve any notable magnitude. In contrast, lower diffusivities concentrate the change in fluid mass, allowing for more substantial stress perturbations as the diffusion front propagates.

A lower bound for the magnitude of stress perturbations can be estimated in the case that the reservoir length and width scale roughly equivalently over time so that the amplitudes of stresses are independent of time (case 2, $\gamma=1$ ). Assuming values of $\mu=$ $10 \mathrm{GPa}, B=0.6, D=1 \mathrm{~km}$, and $\dot{V}=10^{6} \mathrm{~m}^{3} /$ year, the scaling for the distributions in Figs. 3 and 4 would be approximately 3.2 and $32 \mathrm{kPa}$ for $c=1$ and $c=0.1 \mathrm{~m}^{2} / \mathrm{s}$, respectively. If we consider a point at $750 \mathrm{~m}$ depth and $1.75 \mathrm{~km}$ away from the extraction site, the horizontal normal stress perturbation would correspond to a transition from an extensional stress perturbation of $12.8 \mathrm{kPa}$ at $t_{D}=29$ days for $c=0.1 \mathrm{~m}^{2} / \mathrm{s}$ to a compressive perturbation of $-30 \mathrm{kPa}$ after 289 days. For context, such values are roughly an order of magnitude larger than tidal stresses, typically on the order of $1 \mathrm{kPa}$, and $0.1 \%$ of the potential effective confining stresses around $1 \mathrm{~km}$ depth of 15-20 MPa, assuming lithostatic overburden minus hydrostatic pore fluid pressure. The relative significance of such poroelastic perturbations depends heavily on the orientation and existing stress state of faults, including the presence of pore 

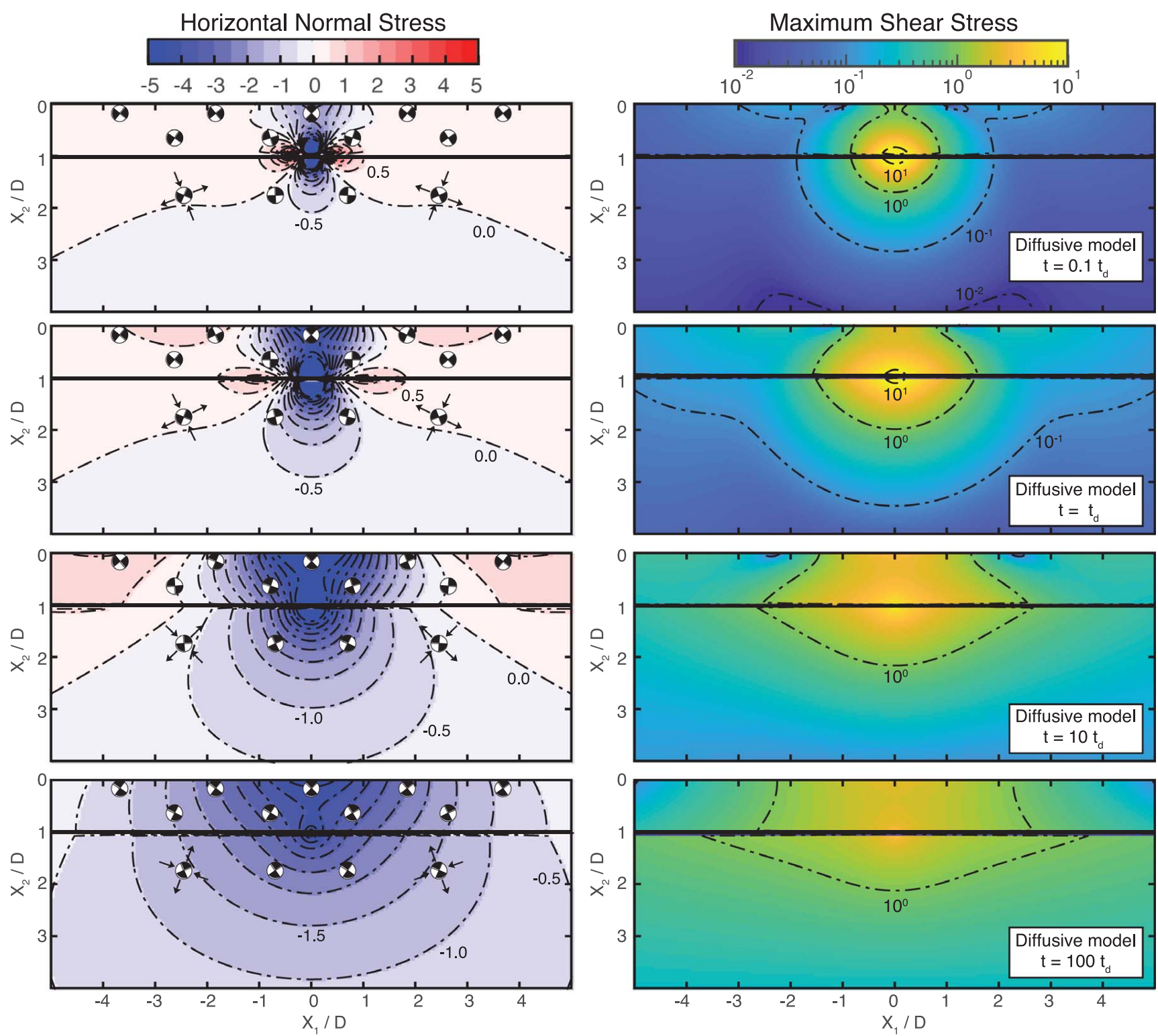

Fig. 3 Change in horizontal normal stress (left) and maximum shear stress (right) due to fluid extraction (relative tension positive) for diffusion models with progressing time relative to the characteristic diffusion timescale $t_{d}=D^{2} / 4 c$. Stresses are contoured outside the producing zone $\left(x_{2} \in[D, D+T]\right)$. Simulated focal mechanisms indicate planes of maximum shear stress, with arrows indicating the axes of maximum tension and compression, and stresses are normalized by $\mu\left(1+v_{u}\right) B T Q / 12 \pi \rho_{0} D\left(1-v_{u}\right) \sqrt{t / c}$.

fluid overpressure within the fault gouge which can substantially alter the effective confining stress acting on faults.

\section{Discussion and Conclusions}

We have expanded upon the analyses presented by Segall $[30,32]$, considering the spatio-temporal evolution of the poroelastic response surrounding fluid extraction from a laterally unconstrained horizontal reservoir. We have examined the implications of allowing for the time-dependent spatial evolution of the effective producing zone due to fluid diffusion, in comparison to assuming a fixed producing geometry. Distinctions arise as the intensity of the poroelastic response is influenced by the spatial gradient of the change in fluid mass content within the reservoir with substantial stress concentrations emerging around sharp gradients in $\Delta m$. Strong gradients in $\Delta m$ would be expected surrounding the extraction site as well as any discontinuities in the reservoir geometry or permeability structure. In the particular case considered here with a uniform diffusivity, sharp gradients would be expected to be alleviated over time due to fluid diffusion, but heterogeneity within the geometric or hydrological structure of the producing layer may result in growing stress concentrations over time as the change in fluid mass within the producing zone increases. A potentially important consideration not incorporated within this model is the possible temporal evolution of the permeability structure within the reservoir, particularly if stresses grow large enough to fracture the surrounding rock. In such a case, there is the possibility that stress concentrations due to structural or permeability irregularities may be smoothed out over time. Overall, the capability for the reservoir to mitigate stress concentrations depends on the hydraulic diffusivity with respect to the volumetric extraction rate where relatively high diffusivities allow for the timely redistribution of pore fluid.

For many reservoir settings, the timescale of fluid transport within the producing layer may be comparable to the timescale for which field observations and seismological studies are made at relevant distances. For these cases, it may be important to 

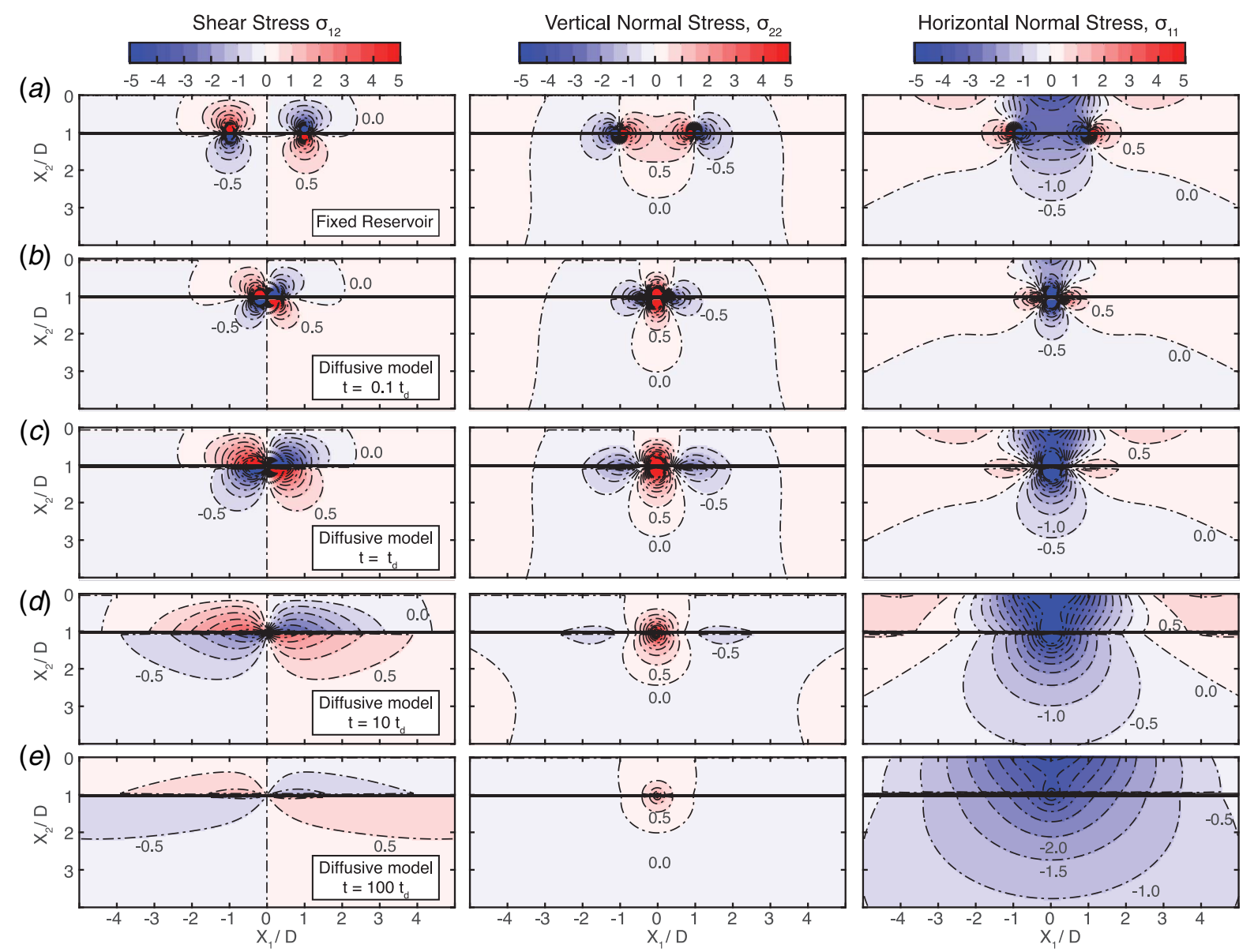

Fig. 4 Extensional and shear stresses due to fluid extraction for diffusion models for progressing time relative to the characteristic diffusion timescale (relative tension positive) in both the (a) fixed reservoir model of Segall [30] and (b)-(e) diffusion models for progressing time relative to the characteristic diffusion timescale. Stresses are normalized for $(a)$ by $\mu\left(1+v_{u}\right) B T Q t / 6 \pi \rho_{0} D^{2}\left(1-v_{u}\right)$ and for $(b)-(e)$ by $\mu\left(1+v_{u}\right) B T Q / 12 \pi \rho_{0} D\left(1-v_{u}\right) \sqrt{t / c}$
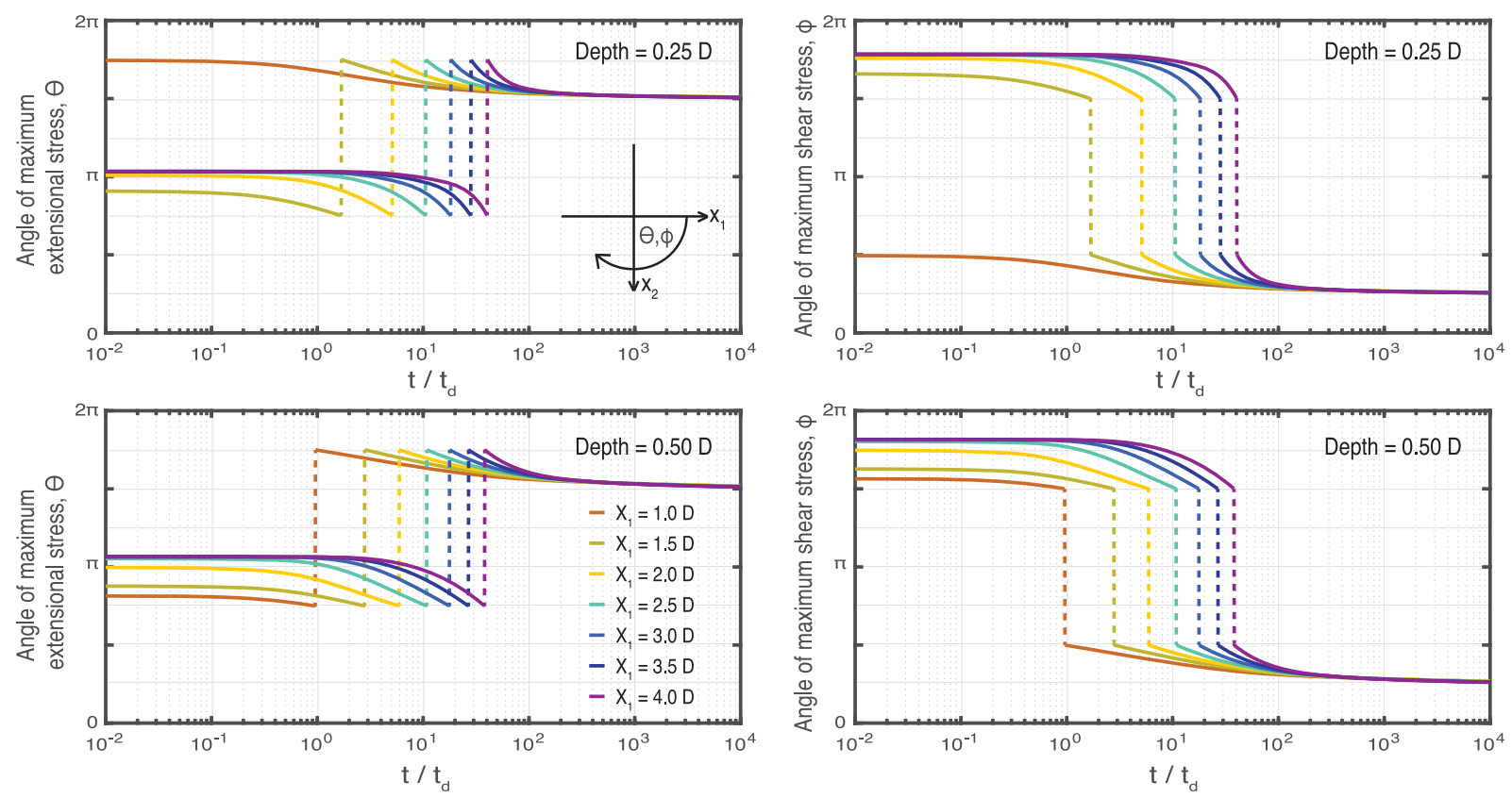

Fig. 5 Temporal evolution of the angle of maximum shear stress $\phi$ (left) and angle of maximum extensional stress $\theta$ (right) as a function of lateral distance from the extraction site at $x_{1}=0$ for representative depths $x_{2}=0.25 D$ (top) and $x_{2}=0.5 D$ (bottom). Note that the rotation of the maximums shear stress angle from $-\pi / 4$ to $\pi / 4$ coincides with the rotation of the horizontal stress from relative tension to compression. 

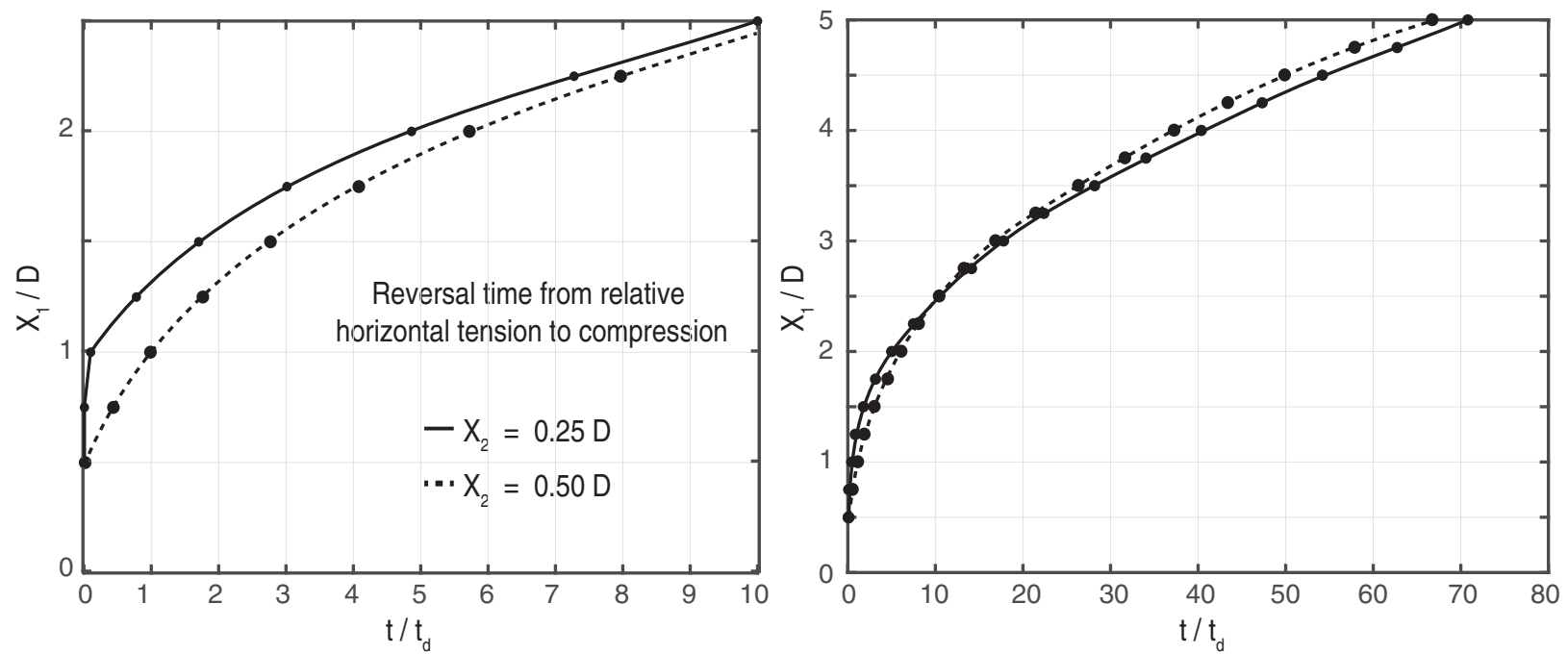

Fig. 6 Lateral spatio-temporal propagation of reversal from relative horizontal compression to tension at two representative depths of $x_{2}=0.25 D$ (solid) and $x_{2}=0.5 D$ (dashed)

account for the nonstationarity of the geometry of the producing zone when interpreting field measurements. Moreover, the magnitude and distribution of surface expressions depend on the balance between the volumetric rate of extraction and the rate at which changes in fluid content can be redistributed through diffusion. Therefore, measurements of the spatio-temporal evolution of surface displacements and strains associated with fluid extraction and injection can allow for the inference of reservoir properties, such as the reservoir diffusivity and thickness.

As introduced by Segall [30], the spatial distribution of stress fields resulting from fluid extraction from a horizontal layer is consistent with reverse faulting above and below the producing zone and normal faulting along the flanks of the reservoir. However, for a laterally unconstrained reservoir, the effective length of the producing zone is not fixed in time and the progression of the producing front away from the extraction site results in a time-dependent rotation of the planes of maximum imposed shear stress as the stress regime transitions from relative horizontal tension to compression above and below the producing layer. This alone may predict that the preferred mechanism of associated seismicity at a given location transitions from normal faulting to reverse faulting as the effective length of the producing zone increases. In contrast, for fluid injection, one may expect a transition from reverse faulting to normal faulting, as inferred for seismicity between 2014 and 2015 in the Crooked Lake and Rocky Mountain House areas in Alberta, Canada, following decades of injection [43]. However, it is important to note that this reflects only a change in polarity of the stress perturbation and not the absolute stress levels around the reservoir, whereas the likelihood of triggered seismicity also depends on the criticality of the pre-existing stress conditions as well as the distribution and orientation of available faults. Rather than a visible change in focal mechanism, the stressing conditions may instead transition from favorable to unfavorable conditions, or vice-versa, depending upon the orientation of existing faults and pre-existing stress state [37]. While general faulting mechanisms may remain consistent, the spatiotemporal evolution of the poroelastic stress field as the effective reservoir expands may be detectable through temporal rotations in the principal stress directions of recorded seismicity, such as those inferred from stress inversions of seismicity in The Geysers geothermal field, California, and the Soultz-sous-Forêts enhanced geothermal system [38-40]. Finally, the stress state surrounding the producing field would also be expected to evolve over time due to stress redistribution from earthquakes as well as aseismic slip within the surrounding region $[16,17,35]$. Overall, developing a better understanding of the spatio-temporal evolution of seismicity and their focal mechanisms may provide additional constraints on the geometry and hydraulic properties of the producing zone $[36,38-40,44]$.

While exploring the conditions for the triggering of seismic and aseismic slip events is beyond the scope of this work, the potential measurement of such temporal transitions in earthquake focal mechanisms or remote activation or deactivation of seismicity may provide constraints on the criticality of regional stress levels. We emphasize the value in the solutions presented by Segall [30-32] and in this work, in providing reference models for which exact calculations may be made for quantitative comparison with field data. Moreover, these solutions may prove useful as reference solutions for more sophisticated numerical methodologies. The $2 \mathrm{D}$ solutions presented here are exact, and we have discussed two approximate extensions to 3D in order to facilitate direct comparison with field measurements and production data, which should be interpreted with care. Finally, while we have focused on the case of fluid extraction where $Q<0$, this analysis may also be considered for the case of fluid injection and diffusion where $Q>0$. Conveniently, the distributions for displacements and stresses will be similar to those shown in this work with only a change in sign due to their linear relationship with the net mass flux $Q$.

\section{Acknowledgment}

Matlab functions for the displacement and stress kernels presented in the Appendix and used in this study can be found on Github. $^{2}$

\section{Nomenclature}

$a=$ horizontal half-width of the reservoir

$c=$ hydraulic diffusivity

$p=$ pore fluid pressure

$q=$ local fluid mass flux

$t=$ time

$u=$ displacement vector

$\mathbf{x}=$ receiver position vector

$\mathbf{y}=$ source position vector

$B=$ Skempton's pore pressure coefficient

$D=$ depth to the top of the reservoir

$Q=$ net mass flux from the producing region

${ }^{2}$ https://github.com/vlambert/ReservoirStresses 


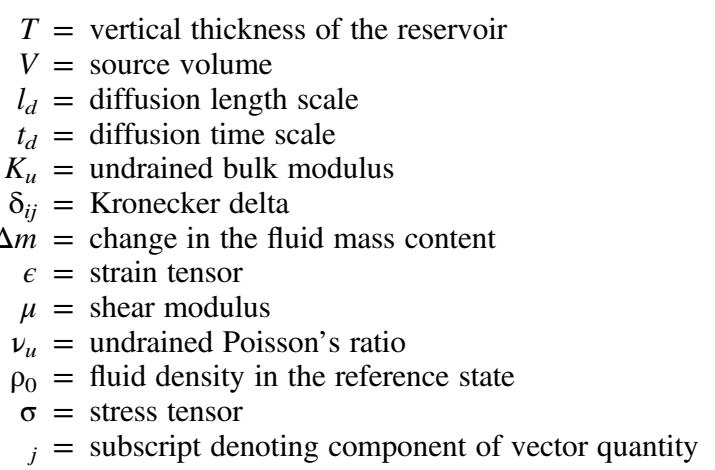

\section{Appendix: Two-Dimensional Poroelastic Solutions}

Segall [32] presented quasi-static solutions for the displacement and stress fields due to changes in the fluid mass content within an infinitesimal volume $d V$ for a 2D linear isotropic poroelastic halfspace. In this study, we calculate these fields resulting from a uniform change in the fluid content given by Eq. (5), as well as the time-dependent distribution resulting from fluid diffusion given by Eq. (7), by convolving the distributions with the poroelastic Green's functions provided in the Appendix of Ref. [32]. Here, we present these solutions again for completeness.

For the general quasi-static problem of fluid extraction from a half plane, the relation for the solid particle displacements can be expressed in the form

$$
u_{j}(\mathbf{x}, t)=\frac{C(\mathbf{y}, t)}{\mu} g_{j}(\mathbf{x}, \mathbf{y})
$$

where $g_{j}(\mathbf{x}, \mathbf{y})$ is the displacement in the $j$ direction at $\mathbf{x}$ due to a point center of dilatation at $\mathbf{y}$ with associated change in the fluid mass content $\Delta m(\mathbf{y}, t) d V$ :

$$
\begin{gathered}
g_{1}(\mathbf{x}, \mathbf{y})=\frac{1}{2}\left[\frac{x_{1}-y_{1}}{r_{1}^{2}(\mathbf{x}, \mathbf{y})}+\frac{\left(3-4 \nu_{u}\right)\left(x_{1}-y_{1}\right)}{r_{2}^{2}(\mathbf{x}, \mathbf{y})}-\frac{4 x_{2}\left(x_{2}+y_{2}\right)\left(x_{1}-y_{1}\right)}{r_{2}^{4}(\mathbf{x}, \mathbf{y})}\right] \\
g_{2}(\mathbf{x}, \mathbf{y})=\frac{1}{2}\left[\frac{x_{2}-y_{2}}{r_{1}^{2}(\mathbf{x}, \mathbf{y})}+\frac{2 x_{2}-\left(3-4 v_{u}\right)\left(x_{2}+y_{2}\right)}{r_{2}^{2}(\mathbf{x}, \mathbf{y})}-\frac{4 x_{2}\left(x_{2}+y_{2}\right)^{2}}{r_{2}^{4}(\mathbf{x}, \mathbf{y})}\right]
\end{gathered}
$$

The prefactor $C(\mathbf{y}, t)=\left(\mu B\left(1+\nu_{u}\right) / 3 \pi \rho_{0}\left(1-\nu_{u}\right)\right) \Delta m(\mathbf{y}, t) d V$ has units of force related to the fluid mass source in the infinitesimal volume $d V$. Here, $u_{1}(\mathbf{x})$ and $u_{2}(\mathbf{x})$ refer to horizontal and vertical displacements, respectively, and $r_{1}^{2}(\mathbf{x}, \mathbf{y})=\left(x_{2}-y_{2}\right)^{2}+\left(x_{1}-y_{1}\right)^{2}$ and $r_{2}^{2}(\mathbf{x}, \mathbf{y})=\left(x_{2}+y_{2}\right)^{2}+\left(x_{1}-y_{1}\right)^{2}$ reflect the source-receiver distances to the true mass source and the image source associated with the free surface at $x_{2}=0$. Note that $\mathbf{y}$ and $\mathbf{x}$ denote the full position vectors for the source and receiver elements, respectively. The poroelastic stresses caused by points of dilatation may also be calculated from Hooke's law in the form $\sigma_{i j}(\mathbf{x}, t)=C(t) G_{i j}(\mathbf{x}, \mathbf{y})$ where

$$
G_{i j}=\frac{\partial g_{i}}{\partial x_{j}}+\frac{\partial g_{j}}{\partial x_{i}}+\frac{2 \nu_{u}}{1-2 \nu_{u}} \frac{\partial g_{k}}{\partial x_{k}} \delta_{i j}
$$

giving [32]

$$
\begin{aligned}
G_{11}(\mathbf{x}, \mathbf{y})= & \frac{\left(x_{2}-y_{2}\right)^{2}-\left(x_{1}-y_{1}\right)^{2}}{r_{1}^{4}(\mathbf{x}, \mathbf{y})}+\frac{\left(x_{2}+y_{2}\right)\left(3 y_{2}-x_{2}\right)-3\left(x_{1}-y_{1}\right)^{2}}{r_{2}^{4}(\mathbf{x}, \mathbf{y})} \\
& +\frac{16 x_{2}\left(x_{2}+y_{2}\right)\left(x_{1}-y_{1}\right)^{2}}{r_{2}^{6}(\mathbf{x}, \mathbf{y})} \\
G_{22}(\mathbf{x}, \mathbf{y})= & \frac{\left(x_{1}-y_{1}\right)^{2}-\left(x_{2}-y_{2}\right)^{2}}{r_{1}^{4}(\mathbf{x}, \mathbf{y})}+\frac{\left(5 x_{2}+y_{2}\right)\left(x_{2}+y_{2}\right)-\left(x_{1}-y_{1}\right)^{2}}{r_{2}^{4}(\mathbf{x}, \mathbf{y})} \\
& -\frac{16 x_{2}\left(x_{2}+y_{2}\right)\left(x_{1}-y_{1}\right)^{2}}{r_{2}^{6}(\mathbf{x}, \mathbf{y})} \\
G_{12}(\mathbf{x}, \mathbf{y})= & -\frac{2\left(x_{1}-y_{1}\right)\left(x_{2}-y_{2}\right)}{r_{1}^{4}(\mathbf{x}, \mathbf{y})}-\frac{2\left(x_{1}-y_{1}\right)\left(3 x_{2}+y_{2}\right)}{r_{2}^{4}(\mathbf{x}, \mathbf{y})} \\
& +\frac{16 x_{2}\left(x_{2}+y_{2}\right)^{2}\left(x_{1}-y_{1}\right)}{r_{2}^{6}(\mathbf{x}, \mathbf{y})}
\end{aligned}
$$

Surface Displacements and Strain Due to Distributed Mass Sources. The total displacement due to changes in a distributed fluid mass at time $t$ can be obtained by integrating the undrained changes in fluid mass within the region for which $\Delta m(\mathbf{y}, t)$ is non-zero according to Eq. (1). Solutions for the surface displacements and horizontal strains resulting from the fixed reservoir mass distribution given by Eq. (5) can be expressed as

$$
\begin{gathered}
u_{1}(\mathbf{x}, t)=\frac{\left(1+\nu_{u}\right) B T Q t}{6 \pi \rho_{0} a} \log \left(\frac{1+\xi_{+}^{2}}{1-\xi_{-}^{2}}\right) \\
u_{2}(\mathbf{x}, t)=\frac{\left(1+\nu_{u}\right) B T Q t}{3 \pi \rho_{0} a}\left[\tan ^{-1}\left(\xi_{-}\right)-\tan ^{-1}\left(\xi_{+}\right)\right] \\
\epsilon_{11}(\mathbf{x}, t)=\frac{\left(1+\nu_{u}\right) B T Q t}{3 \pi \rho_{0} a D}\left[\frac{\xi_{+}}{\xi_{+}^{2}+1}-\frac{\xi_{-}}{\xi_{-}^{2}+1}\right]
\end{gathered}
$$

where $\xi_{-}=x_{1}-a / D$ and $\xi_{+}=x_{1}+a / D$ [30]. For the diffusive source represented by Eq. (7), the corresponding solutions can be expressed as Eqs. (A11)-(A13), where $M\left(y_{1}, t\right)=\left[(1 / \sqrt{\pi}) \exp \left(-y_{1}^{2} / 4 c t\right)-\left|y_{1}\right| / 2 \sqrt{c t} \operatorname{erfc}\left(\left|y_{1}\right| / 2 \sqrt{c t}\right)\right]$. The integral over $y_{1}$ can be computed numerically for the diffusive source.

$$
\begin{gathered}
u_{1}(\mathbf{x}, t)=\frac{2\left(1+\nu_{u}\right) B Q}{3 \pi \rho_{0}} \sqrt{\frac{t}{c}} \int M\left(y_{1}, t\right)\left(\tan ^{-1}\left(\frac{D+T}{x_{1}-y_{1}}\right)-\tan ^{-1}\left(\frac{D}{x_{1}-y_{1}}\right)\right) d y_{1} \\
u_{2}(\mathbf{x}, t)=\frac{\left(1+v_{u}\right) B Q}{3 \pi \rho_{0}} \sqrt{\frac{t}{c}} \int M\left(y_{1}, t\right) \log \left(\frac{\left.\left(x_{1}-y_{1}\right)^{2}+D^{2}\right)}{\left(x_{1}-y_{1}\right)^{2}+(D+T)^{2}}\right) d y_{1}
\end{gathered}
$$




$$
\epsilon_{11}(\mathbf{x}, t)=\frac{\left(1+\nu_{u}\right) T B Q}{3 \pi \rho_{0}} \sqrt{\frac{t}{c}} \int M\left(y_{1}, t\right)\left(\frac{D(D+T)-\left(x_{1}-y_{1}\right)^{2}}{\left(D^{2}+\left(x_{1}-y_{1}\right)^{2}\right)\left((D+T)^{2}+\left(x_{1}-y_{1}\right)^{2}\right)}\right) d y_{1}
$$

Stresses Due to Distributed Mass Sources. The stress distributions resulting from the distributed source may also be represented through linear integral relations with the poroelastic Green's functions $G_{n m}(\mathbf{x}, \mathbf{y})$. By considering the stress distributions only outside of the reservoir, where $\Delta m(\mathbf{y}, t)=0$, these expressions simplify to the form of Eq. (8). Fully analytical expressions for the stress fields associated with the fixed reservoir mass distribution represented by Eq. (5) can be solved as

$$
\sigma_{i j}(\mathbf{x}, t)=\frac{\mu\left(1+v_{u}\right) B}{3 \pi \rho_{0}\left(1-v_{u}\right)} \int_{V} \frac{Q t}{2 a} G_{i j}(\mathbf{x}, \mathbf{y}) d V_{\mathrm{y}}=\frac{\mu\left(1+\nu_{u}\right) B Q t}{6 \pi \rho_{0} a\left(1-v_{u}\right)} \int_{D}^{D+T} \int_{-a}^{a} G_{i j}\left(x_{1}, x_{2} ; y_{1}, y_{2}\right) d y_{1} d y_{2}
$$

These solutions are separable in space and time and can be expressed as $\sigma_{i j}(\mathbf{x}, t)=\left(\mu\left(1+\nu_{u}\right) B Q t / 6 \pi \rho_{0} a\left(1-\nu_{u}\right)\right) K_{i j}(\mathbf{x})$, where the kernels $K_{i j}$ are expressed as Eqs. (A15)-(A17) [30].

$$
\begin{aligned}
K_{11}(\mathbf{x})= & \tan ^{-1}\left(\frac{x_{2}-(D+T)}{x_{1}-a}\right)-\tan ^{-1}\left(\frac{x_{2}-(D+T)}{x_{1}+a}\right)+\tan ^{-1}\left(\frac{x_{2}-D}{x_{1}+a}\right)-\tan ^{-1}\left(\frac{x_{2}-D}{x_{1}-a}\right) \\
& +3\left(\tan ^{-1}\left(\frac{x_{2}+(D+T)}{x_{1}+a}\right)-\tan ^{-1}\left(\frac{x_{2}+(D+T)}{x_{1}-a}\right)+\tan ^{-1}\left(\frac{x_{2}+D}{x_{1}-a}\right)-\tan ^{-1}\left(\frac{x_{2}+D}{x_{1}+a}\right)\right) \\
& +4 a x_{2}\left(\frac{a^{2}-x_{1}^{2}+\left(x_{2}+D+T\right)^{2}}{\left(\left(x_{1}-a\right)^{2}+\left(x_{2}+D+T\right)^{2}\right)\left(\left(x_{1}+a\right)^{2}+\left(x_{2}+D+T\right)^{2}\right)}-\frac{a^{2}}{\left(\left(x_{1}-a\right)^{2}+\left(x_{2}+D\right)^{2}\right)\left(\left(x_{1}+a\right)^{2}+\left(x_{2}+D\right)^{2}\right)}\right) \\
K_{22}(\mathbf{x})= & \tan ^{-1}\left(\frac{x_{2}-(D+T)}{x_{1}+a}\right)-\tan ^{-1}\left(\frac{x_{2}-(D+T)}{x_{1}-a}\right)+\tan ^{-1}\left(\frac{x_{2}-D}{x_{1}-a}\right)-\tan ^{-1}\left(\frac{x_{2}-D}{x_{1}+a}\right) \\
& +\tan ^{-1}\left(\frac{x_{2}+(D+T)}{x_{1}+a}\right)-\tan ^{-1}\left(\frac{x_{2}+(D+T)}{x_{1}-a}\right)+\tan ^{-1}\left(\frac{x_{2}+D}{x_{1}-a}\right)-\tan ^{-1}\left(\frac{x_{2}+D}{x_{1}+a}\right) \\
& +4 a x_{2}\left(\frac{a^{2}-x_{1}^{2}+\left(x_{2}+D+T\right)^{2}}{\left(\left(x_{1}-a\right)^{2}+\left(x_{2}+D\right)^{2}\right)\left(\left(x_{1}+a\right)^{2}+\left(x_{2}+D\right)^{2}\right)}-\frac{\left(\left(x_{1}-a\right)^{2}+\left(x_{2}+D+T\right)^{2}\right)\left(\left(x_{1}+a\right)^{2}+\left(x_{2}+D+T\right)^{2}\right)}{(2)}\right)
\end{aligned}
$$

$$
\begin{aligned}
K_{12}(\mathbf{x})= & \frac{1}{2}\left[\log \left(\left(x_{1}-a\right)^{2}+\left(x_{2}-(D+T)\right)^{2}\right)-\log \left(\left(x_{1}-a\right)^{2}+\left(x_{2}-D\right)^{2}\right)\right. \\
& +\frac{16 a x_{1} x_{2}\left(x_{2}+D\right)}{\left(\left(x_{1}-a\right)^{2}+\left(x_{2}+D\right)^{2}\right)\left(\left(x_{1}+a\right)^{2}+\left(x_{2}+D\right)^{2}\right)}-\frac{16 a x_{1} x_{2}\left(x_{2}+D+T\right)}{\left(\left(x_{1}-a\right)^{2}+\left(x_{2}+D+T\right)^{2}\right)\left(\left(x_{1}+a\right)^{2}+\left(x_{2}+D+T\right)^{2}\right)} \\
& +\left(\left(x_{1}-a\right)^{2}+\left(x_{2}-D\right)^{2}\right)\left(\left(x_{1}+a\right)^{2}+\left(x_{2}+D\right)^{2}\right)\left(\frac{\log \left(\left(x_{1}+a\right)^{2}+\left(x_{2}-D\right)^{2}\right)}{\left(\left(x_{1}-a\right)^{2}+\left(x_{2}+D\right)^{2}\right)\left(\left(x_{1}+a\right)^{2}+\left(x_{2}+D\right)^{2}\right)}\right. \\
& \left.+\frac{\log \left(\left(x_{1}-a\right)^{2}+\left(x_{2}+D\right)^{2}\right)}{\left(\left(x_{1}-a\right)^{2}+\left(x_{2}+D\right)^{2}\right)\left(\left(x_{1}+a\right)^{2}+\left(x_{2}+D\right)^{2}\right)}-\frac{\left.\log +D)^{2}\right)}{\left(\left(x_{1}-a\right)^{2}+\left(x_{2}+D\right)^{2}\right)\left(\left(x_{1}+a\right)^{2}+\left(x_{2}+D\right)^{2}\right)}\right) \\
& -\left(\left(x_{1}-a\right)^{2}+\left(x_{2}-D-T\right)^{2}\right)\left(\left(x_{1}+a\right)^{2}+\left(x_{2}+D+T\right)^{2}\right) \\
& \times\left(\frac{\log \left(\left(x_{1}+a\right)^{2}+\left(x_{2}-D-T\right)^{2}\right)}{\left(\left(x_{1}-a\right)^{2}+\left(x_{2}+D+T\right)^{2}\right)\left(\left(x_{1}+a\right)^{2}+\left(x_{2}+D+T\right)^{2}\right)}+\frac{\left.\log +T)^{2}\right)}{\left(\left(x_{1}-a\right)^{2}+\left(x_{2}+D+T\right)^{2}\right)\left(\left(x_{1}+a\right)^{2}+\left(x_{2}+D+T\right)^{2}\right)}\right. \\
& \left.\left.-\frac{\log \left(\left(x_{1}+a\right)^{2}+\left(x_{2}+D+T\right)^{2}\right)}{\left(\left(x_{1}-a\right)^{2}+\left(x_{2}+D+T\right)^{2}\right)\left(\left(x_{1}+a\right)^{2}+\left(x_{2}+D+T\right)^{2}\right)}\right)\right]
\end{aligned}
$$

Solutions resulting from the diffusive mass source represented by Eq. (7) can be expressed similarly, however, the spatio-temporal evolution of the stress fields are coupled due to the time-dependence of the fluid diffusion process

$$
\sigma_{i j}(\mathbf{x}, t)=C \int_{V} Q\left(\frac{t}{c}\right)^{1 / 2} \operatorname{ierfc}\left[\left(\frac{y_{1}^{2}}{4 c t}\right)^{1 / 2}\right] G_{i j}(\mathbf{x}, \mathbf{y}) d V_{\mathbf{y}}=C Q\left(\frac{t}{c}\right)^{1 / 2} \int_{D}^{D+T} \int_{-\infty}^{\infty} \operatorname{ierfc}\left[\left(\frac{y_{1}^{2}}{4 c t}\right)^{1 / 2}\right] G_{i j}\left(x_{1}, x_{2} ; y_{1}, y_{2}\right) d y_{1} d y_{2}
$$

where $C=\mu\left(1+\nu_{u}\right) B / 3 \pi \rho_{0}\left(1-\nu_{u}\right)$. Individual stress components can be expressed as $\sigma_{i j}(\mathbf{x}, \mathbf{y}, t)=\mu\left(1+\nu_{u}\right) B Q / 3 \pi \rho_{0}\left(1-\nu_{u}\right)(t / c)^{1 / 2} J_{i j}(\mathbf{x}, \mathbf{y}$, $t$ ), where the kernels $J_{i j}$ expressed in Eqs. (A18)-(A19) can be computed numerically.

$$
\begin{aligned}
J_{11}(\mathbf{x}, \mathbf{y}, t)= & \int M\left(y_{1}, t\right)\left[\frac{x_{2}+3 D}{\left(x_{1}-y_{1}\right)^{2}+\left(x_{2}+D\right)^{2}}+\frac{4 x_{2}\left(x_{1}-y_{1}\right)^{2}}{\left(x_{1}-y_{1}\right)^{2}+\left(x_{2}+D\right)^{2}}-\frac{x_{2}-D}{\left(x_{1}-y_{1}\right)^{2}+\left(x_{2}-D\right)^{2}}\right. \\
& \left.-\frac{x_{2}+3(D+T)}{\left(x_{1}-y_{1}\right)^{2}-\left(x_{2}+D+T\right)^{2}}-\frac{4 x_{2}\left(x_{1}-y_{1}\right)^{2}}{\left(x_{1}-y_{1}\right)^{2}+\left(x_{2}+D+T\right)^{2}}+\frac{x_{2}-D+T}{\left(x_{1}-y_{1}\right)^{2}+\left(x_{2}-D-T\right)^{2}}\right] d y_{1}
\end{aligned}
$$




$$
\begin{gathered}
J_{22}(\mathbf{x}, \mathbf{y}, t)=4 x_{2}^{2} \int M\left(y_{1}, t\right)\left[\frac{D\left(x_{2}^{2}+3\left(x_{1}-y_{1}\right)^{2}\right)+x_{2}\left(x_{2}^{2}+\left(x_{1}-y_{1}\right)^{2}\right)-x_{2} D^{2}-D^{3}}{\left(\left(x_{1}-y_{1}\right)^{2}+\left(x_{2}-D\right)^{2}\right)\left(\left(x_{1}-y_{1}\right)^{2}+\left(x_{2}+D\right)^{2}\right)^{2}}\right. \\
\left.-\frac{(D+T)\left(x_{2}^{2}+3\left(x_{1}-y_{1}\right)^{2}\right)+x_{2}\left(x_{2}^{2}+\left(x_{1}-y_{1}\right)^{2}\right)-x_{2}(D+T)^{2}-(D+T)^{3}}{\left(\left(x_{1}-y_{1}\right)^{2}+\left(x_{2}-D-T\right)^{2}\right)\left(\left(x_{1}-y_{1}\right)^{2}+\left(x_{2}+D+T\right)^{2}\right)^{2}}\right] d y_{1} \\
J_{12}(\mathbf{x}, \mathbf{y}, t)= \\
4 x_{2} \int\left(x_{1}-y_{1}\right) M\left(y_{1}, t\right)\left[\frac{2 D\left(x_{1}-y_{1}\right)^{2}+x_{2}\left(x_{2}^{2}+\left(x_{1}-y_{1}\right)^{2}\right)+x_{2} D^{2}+2 D^{3}}{\left(\left(x_{1}-y_{1}\right)^{2}+\left(x_{2}-D\right)^{2}\right)\left(\left(x_{1}-y_{1}\right)^{2}+\left(x_{2}+D\right)^{2}\right)^{2}}\right. \\
\left.-\frac{2(D+T)\left(x_{1}-y_{1}\right)^{2}+x_{2}\left(x_{2}^{2}+\left(x_{1}-y_{1}\right)^{2}\right)+x_{2}(D+T)^{2}+2(D+T)^{3}}{\left(\left(x_{1}-y_{1}\right)^{2}+\left(x_{2}-D-T\right)^{2}\right)\left(\left(x_{1}-y_{1}\right)^{2}+\left(x_{2}+D+T\right)^{2}\right)^{2}}\right] d y_{1}
\end{gathered}
$$
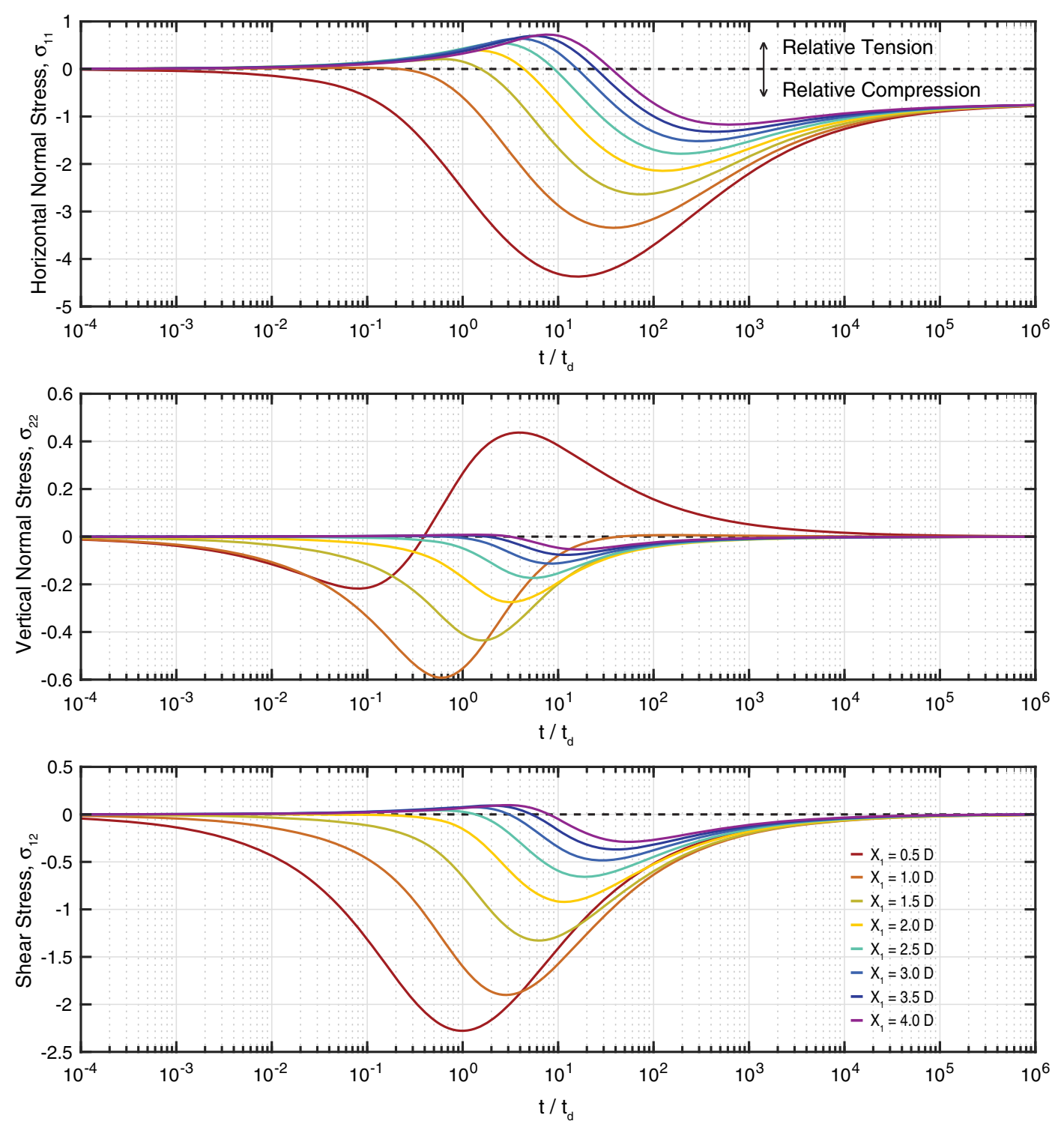

Fig. 7 Temporal evolution of the horizontal $\sigma_{11}$ and vertical $\sigma_{22}$ normal stresses and shear stress $\sigma_{12}$ as a function of lateral distance from the extraction site at $x_{1}=0$ for the representative depth $x_{2}=0.25 D$. Stresses are normalized by $\mu\left(1+v_{u}\right) B T Q / 12 \pi \rho_{0} D\left(1-v_{u}\right) \sqrt{t / c}$.

The spatial distribution of the horizontal and vertical normal stresses, $\sigma_{11}$ and $\sigma_{22}$, respectively, as well as the shear stress $\sigma_{12}$ for the fixed reservoir and diffusive models is shown in Fig. 4. The temporal evolution of these three stress components as a function of lateral distance from the extraction site is shown in Fig. 7, where one can note the transition between relative horizontal tension to compression at each site. The amplitudes of the associated principal stresses and maximum shear stresses are given in 

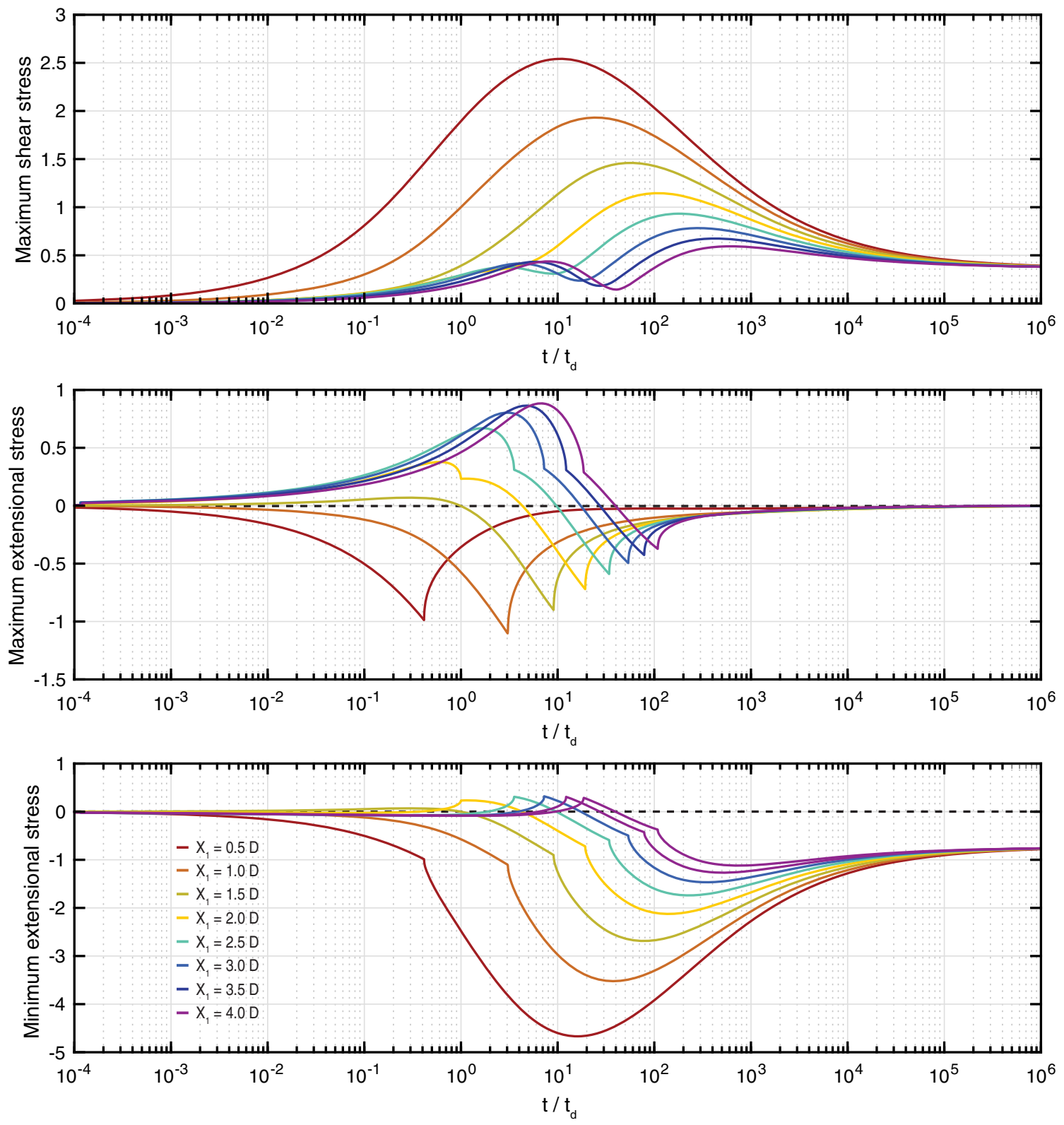

Fig. 8 Temporal evolution of the maximum shear stress and principal stresses as a function of lateral distance from the extraction site at $x_{1}=0$ for the representative depth $x_{2}=0.25 D$. Stresses are normalized by $\mu\left(1+v_{u}\right) B T Q /$ $12 \pi \rho_{0} D\left(1-v_{u}\right) \sqrt{t / c}$.

Fig. 8. For distances beyond $x_{1}=2 D$, an inflection is noticeable in the maximum shear stress which coincides with the transition from relative horizontal tension to compression. The lateral progression of the reversal front to relative compression is shown in Fig. 6 for representative depths of $x_{2}=0.25 \mathrm{D}$ and $x_{2}=0.5 \mathrm{D}$. As the effective producing zone expands as $l_{d} \propto \sqrt{t}$, the propagation of the reversal front is faster early on at locations closer to the extraction point but decreases at further distances.

\section{References}

[1] Zoback, M., 2013, "Managing the Seismic Risk Posed by Wastewater Disposal," Earth Mag., 57, pp. 38-42.

[2] Ellsworth, W. L., 2013, "Injection-Induced Earthquakes," Science, 341(6142), p. 1225942.

[3] McGarr, A., Bekins, B., Burkardt, N., Dewey, J., Earle, P., Ellsworth, W., Ge, S., Hickman, S., Holland, A., Majer, E., Rubinstein, J., and Sheehan, A., 2015,
"Coping With Earthquakes Induced by Fluid Injection," Science, 347(6224), pp. 830-831.

[4] Lee, K.-K., Ellsworth, W. L., Giardini, D., Townend, J., Ge, S., Shimamoto, T., Yeo, I.-W., Kang, T.-S., Rhie, J., Sheen, D.-H., Chang, C., Woo, J.-U., and Langenbruch, C., 2019, "Managing Injection-Induced Seismic Risks," Science, 364(6442), pp. 730-732.

[5] Healy, J. H., Rubey, W. W., Griggs, D. T., and Raleigh, C. B., 1968, "The Denver Earthquakes," Science, 161(3848), pp. 1301-1310.

[6] Raleigh, C. B., Healy, J. H., and Bredehoeft, J. D., 2013, Faulting and Crustal Stress at Rangely, Colorado. In Flow and Fracture of Rocks, American Geophysical Union, pp. 275-284.

[7] Raleigh, C. B., Healy, J. H., and Bredehoeft, J. D., 1976, “An Experiment in Earthquake Control at Rangely, Colorado," Science, 191(4233), pp. 1230-1237.

[8] Hsieh, P. A., and Bredehoeft, J. D., 1981, "A Reservoir Analysis of the Denver Earthquakes: A Case of Induced Seismicity," J. Geophys. Res. Solid Earth, 86(B2), pp. 903-920.

[9] Frohlich, C., Hayward, C., Stump, B., and Potter, E., 2011, "The Dallas-Fort Worth Earthquake Sequence: October 2008 Through May 2009," Bull. Seismol. Soc. Am., 101(1), pp. 327-340.

[10] Horton, S., 2012, "Disposal of Hydrofracking Waste Fluid by Injection Into Subsurface Aquifers Triggers Earthquake Swarm in Central Arkansas With Potential for Damaging Earthquake," Seismol. Res. Lett., 83(2), pp. 250-260. 
[11] Kim, W.-Y., 2013, “Induced Seismicity Associated With Fluid Injection Into a Deep Well in Youngstown, Ohio," J. Geophys. Res. Solid Earth, 118(7), pp. 3506-3518.

[12] Zhang, Y., Person, M., Rupp, J., Ellett, K., Celia, M. A., Gable, C. W., Bowen, B., Evans, J., Bandilla, K., Mozley, P., Dewers, T., and Elliot, T., 2013, "Hydrogeologic Controls on Induced Seismicity In-Crystalline Basement Rocks Due to Fluid Injection Into Basal Reservoirs," Groundwater, 51(4), pp. 525-538.

[13] Frohlich, C., Ellsworth, W., Brown, W. A., Brunt, M., Luetgert, J., MacDonald, T., and Walter, S., 2014, "The 17 May 2012 M4.8 Earthquake Near Timpson, East Texas: An Event Possibly Triggered by Fluid Injection,” J. Geophys. Res. Solid Earth, 119(1), pp. 581-593.

[14] Walsh, F., and Zoback, M. D., 2015, "Oklahoma's Recent Earthquakes and Salt Water Disposal," Sci. Adv., 1(5), p. e1500195.

[15] Keranen, K. M., Weingarten, M., Abers, G. A., Bekins, B. A., and Ge, S., 2014, "Sharp Increase in Central Oklahoma Seismicity Since 2008 Induced by Massive Wastewater Injection," Science, 345(6195), pp. 448-451.

[16] Guglielmi, Y., and Elsworth, D., 2015, "Seismicity Triggered by Fluid Injection-Induced Aseismic Slip," Science, 348(6240), pp. 1224-1226.

[17] Bhattacharya, P., and Viesca, R. C., 2019, "Fluid-Induced Aseismic Fault Slip Outpaces Pore-Fluid Migration," Science, 364(6439), pp. 464-468.

[18] Yerkes, R., and Castle, R., 1970, "Surface Deformation Associated With Oil and Gas Field Operations in the United States, in Land Subsidencel," Int. Assoc. Sci. Hydrol. UNESCO Publ., 89(1), pp. 55-66.

[19] Pennington, W. D., Davis, S. D., Carlson, S. M., DuPree, J., and Ewing, T. E., 1986, "The Evolution of Seismic Barriers and Asperities Caused by the Depressuring of Fault Planes in Oil and Gas Fields of South Texas," Bull. Seismol. Soc. Am., 76(4), pp. 939-948.

[20] Wetmiller, R. J., 1986, "Earthquakes Near Rocky Mountain House, Alberta, and Their Relationship to Gas Production Facilities," Can. J. Earth Sci., 23(2), pp. 172-181.

[21] Grasso, J. R., and Wittlinger, G., 1990, "Ten Years of Seismic Monitoring Over a Gas Field," Bull. Seismol. Soc. Am., 80(2), pp. 450-473.

[22] Feignier, B., and Grasso, J., 1990, "Seismicity Induced by a Gas-Production: I. Correlation of Focal Mechanisms and Dome Structure," Pure Appl. Geophys., 134(3), pp. 405-426.

[23] Grasso, J. R., and Feignier, B., 1989, "Seismicity Induced by Gas-Production: 2. Lithology Correlated Events, Induced Stresses and Deformation," Pure Appl. Geophys., 134, pp. 427-450.

[24] Doser, D. I., Baker, M. R., and Mason, D. B., 1991, "Seismicity in the War-Wink Gas Field, Delaware Basin, West Texas, and Its Relationship to Petroleum Production,” Bull. Seismol. Soc. Am., 81(3), pp. 971-986.

[25] Guyoton, F., Grasso, J.-R., and Volant, P., 1992, "Interrelation Between Induced Seismic Instabilities and Complex Geological Structure," Geophys. Res. Lett., 19(7), pp. 705-708.

[26] Segall, P., Grasso, J.-R., and Mossop, A., 1994, "Poroelastic Stressing and Induced Seismicity Near the lacq Gas Field, Southwestern France," J. Geophys. Res. Solid Earth, 99(B8), pp. 15423-15438.

[27] Frohlich, C., DeShon, H., Stump, B., Hayward, C., Hornbach, M., and Walter, J., 2016, "A Historical Review of Induced Earthquakes in Texas," Seismol. Rev. Lett., 87(4), pp. 1022-1038.
[28] Hough, S. E., Tsai, V. C., Walker, R., and Aminzadeh, F., 2017, "Was the mw 7.5 1952 kern County, California, Earthquake Induced (or Triggered)?” J. Seismol., 21(6), pp. 1613-1621.

[29] Hough, S. E., and Bilham, R., 2018, "Revisiting Earthquakes in the Los Angeles, California, Basin During the Early Instrumental Period: Evidence for an Association With Oil Production," J. Geophys. Res. Solid Earth, 123(12), pp. 10684-10705.

[30] Segall, P., 1989, "Earthquakes Triggered by Fluid Extraction," Geology, 17(10), pp. 942-946.

[31] Segall, P., 1992, "Induced Stresses Due to Fluid Extraction From Axisymmetric Reservoirs," Pure Appl. Geophys., 139, pp. 535-560.

[32] Segall, P., 1985, "Stress and Subsidence Resulting From Subsurface Fluid Withdrawal in the Epicentral Region of the 1983 Coalinga Earthquake," J. Geophys. Res. Solid Earth, 90(B8), pp. 6801-6816.

[33] Keranen, K. M., and Weingarten, M., 2018, "Induced Seismicity," Annu. Rev. Earth Planet Sci., 46(1), pp. 149-174.

[34] Smith, J. D., Avouac, J.-P., White, R. S., Copley, A., Gualandi, A., and Bourne, S., 2019, "Reconciling the Long-Term Relationship Between Reservoir Pore Pressure Depletion and Compaction in the Groningen Region," J. Geophys. Res. Solid Earth, 124(6), pp. 6165-6178.

[35] Keranen, K. M., Savage, H. M., Abers, G. A., and Cochran, E. S., 2013, "Potentially Induced Earthquakes in Oklahoma, USA: Links Between Wastewater Injection and the 2011 Mw 5.7 Earthquake Sequence," Geology, 41(6), pp. 699-702.

[36] Mukuhira, Y., Fuse, K., Naoi, M., Fehler, M., Moriya, H., Ito, T., Asanuma, H., and Häring, M., 2018, "Hybrid Focal Mechanism Determination: Constraining Focal Mechanisms of Injection Induced Seismicity Using In Situ Stress Data," Geophys. J. Int., 215(2), pp. 1427-1441.

[37] Segall, P., and Lu, S., 2015, "Injection-Induced Seismicity: Poroelastic and Earthquake Nucleation Effects," J. Geophys. Res. Solid Earth, 120(7), pp. 5082-5103.

[38] Martínez-Garzón, P., Bohnhoff, M., Kwiatek, G., and Dresen, G., 2013, "Stress Tensor Changes Related to Fluid Injection at the Geysers Geothermal Field, California," Geophys. Res. Lett., 40(11), pp. 2596-2601.

[39] Martínez-Garzón, P., Kwiatek, G., Sone, H., Bohnhoff, M., Dresen, G., and Hartline, C., 2014, "Spatiotemporal Changes, Faulting Regimes, and Source Parameters of Induced Seismicity: A Case Study From the Geysers Geothermal Field," J. Geophys. Res. Solid Earth, 119(11), pp. 8378-8396.

[40] Schoenball, M., Dorbath, L., Gaucher, E., Wellmann, J. F., and Kohl, T., 2014, "Change of Stress Regime During Geothermal Reservoir Stimulation," Geophys. Res. Lett., 41(4), pp. 1163-1170.

[41] Nur, A., and Byerlee, J. D., 1971, "An Exact Effective Stress Law for Elastic Deformation of Rock With Fluids," J. Geophys. Res. (1896-1977), 76(26), pp. 6414-6419.

[42] Carslaw, A. R., and Jaeger, C. J., 1959, Conduction of Heat in Solids, Oxford Science Publications, Oxford.

[43] Eaton, D. W., and Mahani, A. B., 2015, "Focal Mechanisms of Some Inferred Induced Earthquakes in Alberta, Canada," Seismol. Res. Lett., 86(4), pp. 10781085 .

[44] Shapiro, S., Rothert, E., Rath, V., and Rindschwentner, J., 2002, "Characterization of Fluid Transport Properties of Reservoirs Using Induced Microseismicity," Geophysics, 67(1), pp. 14-26. 